\title{
Simultaneous control of natural and extra degrees of freedom by isometric force and electromyographic activity in the muscle-to-force null space
}

This is a pre print version of the following article:

Original:

Gurgone, S., Borzelli, D., de Pasquale, P., Berger, D.J., Lisini Baldi, T., D'Aurizio, N., et al. (2022). Simultaneous control of natural and extra degrees of freedom by isometric force and electromyographic activity in the muscle-to-force null space. JOURNAL OF NEURAL ENGINEERING, 19(1) [10.1088/1741$2552 / a c 47 d b]$.

Availability:

This version is availablehttp://hdl.handle.net/11365/1184023

since 2022-02-05T16:52:09Z

\section{Published:}

DOI:10.1088/1741-2552/ac47db

Terms of use:

Open Access

The terms and conditions for the reuse of this version of the manuscript are specified in the publishing policy. Works made available under a Creative Commons license can be used according to the terms and conditions of said license.

For all terms of use and more information see the publisher's website.

(Article begins on next page) 


\title{
Simultaneous control of natural and extra degrees of freedom by isometric force and electromyographic activity in the muscle-to-force null space
}

\author{
Sergio Gurgone ${ }^{1}$, Daniele Borzelli 2,3 , Paolo De Pasquale 2,3 , Denise Jennifer Berger ${ }^{3,4}$, Tommaso Lisini Baldi ${ }^{5}$, Nicole $D^{\prime}$ Aurizio $^{5}$, \\ Domenico Prattichizzo 5,6 , Andrea d'Avella2,3 \\ 1Department of Matemathical and Computer Sciences, Physical Sciences and Earth Sciences, University of Messina, Messina (ME), Italy \\ 2 Department of Biomedical, Dental, Morphological and Functional Imaging Sciences, University of Messina, Messina (ME), Italy \\ 3 Laboratory of Neuromotor Physiology, IRCCS Fondazione Santa Lucia, Rome, Italy \\ 4 Department of Systems Medicine and Centre of Space Bio-medicine, University of Rome Tor Vergata, Rome, Italy \\ 5 Department of Information Engineering and Mathematics, University of Siena, Siena, Italy \\ 6 Humanoids and Human Centered Mechatronics, Istituto Italiano di Tecnologia, Genoa, Italy
}

\begin{abstract}
Objective. Muscle activation patterns in the muscle-to-force null space may provide an opportunity for motor augmentation by allowing to control additional end-effectors simultaneously to natural limbs. Here we tested the feasibility of muscular null space control for augmentation by assessing simultaneous control of natural and extra degrees of freedom (DoFs). Approach. We instructed eight participants to control translation and rotation of a virtual 3D end-effector by simultaneous generation of isometric force at the hand and null space activity extracted in real-time from the electromyographic signals recorded from 15 shoulder and arm muscles. First, we identified the null space components that each participant could control more naturally by voluntary co-contraction. Then, participants performed several blocks of a reaching and holding task. They displaced an ellipsoidal cursor to reach one of nine targets by generating force, and simultaneously rotated the cursor to match the target orientation by activating null space components. We developed a information-theoretic metric, inspired by Fitts' law, to assess individual null space control ability. We introduced an index of difficulty (ID) as the sum of a spatial ID for target reaching and a temporal ID for target holding. Main Results. On average, participants could reach the targets in most trials already in the first block (72\%) and they improved with practice (93\% in the final block) but holding performance remained lower ( $43 \%$ in the final block). Remarkably, there was a high interindividual variability in performance, and the ID computed in a simulation with different spatial and temporal task conditions allowed to estimate those for which each individual participants could perform best. Significance. Muscular null space control is feasible and may be used to control additional virtual or robotics end-effectors. However, decoding of motor commands must be optimized according to individual null space control ability.
\end{abstract}

Keywords: electromyography, muscle-to-force null space, human augmentation, myoelectric control, virtual reality, reaching, Fitts' law

\section{Introduction}

Electromyographic (EMG) signals have been used for many years to control upper and lower limb prostheses [1-6], rehabilitation robotic devices [7-11], and virtual end-effectors [12-15]. Myoelectric control of a prosthetic limb by an amputee, as a replacement of the missing limb, relies either on the detection of movement intention by EMG pattern recognition $[16,17]$ or on the direct control of one or multiple 
degrees of freedom using EMG signals recorded from many different muscles $[4,18,19]$. EMG signals have long been used for the control of robotic devices such as exoskeletons [20]. Differently from prostheses, exoskeletons can assist both ablebodied and disabled people, enhancing existing abilities [21] or substituting missing ones [22], and can be used for rehabilitation after orthopedic or neurological injuries [2325]. Myoelectric control is also a powerful tool to investigate basic principles of human motor control [12-14]. By using EMG signals to control a cursor in a virtual environment, it is possible to alter the mapping between motor commands and end-effector motion and to study how the central nervous system adapts to such perturbations. For example, a linear mapping of EMG signals onto isometric end-point forces applied to a simulated mass can be altered ("virtual surgery") such that new muscle synergies are required to compensate the perturbation [12]. Thus, to date, myoelectric control has been used mostly either to control an external device or to assist the movement of a natural limb.

Myoelectric control, however, could also be used to control an external device concurrently with the motion of the natural limbs, possibly augmenting human motor capabilities. At the basis of augmentation lies the concept of motor task null space. Due to the redundancy of the musculoskeletal and neural systems, i.e. the presence of a higher number of active units (muscles and neurons) than the end-effector degrees of freedom involved in a task, many combinations of joint angles, muscle patterns and neural signals do not generate task-relevant movements or forces [26]. Such combinations lie in the kinematic, muscular and neural null space respectively. A few different approaches for augmentation based on these concepts have been recently investigated. Abdi and collaborators [27] developed three-handed manipulation in a virtual environment, using the motion of a foot to control the third hand in a simple task rather than muscle activity. Similarly, a third robotic thumb controlled using a toe [28] and a sixth finger controlled through kinematic null space of upper limbs [29,30] have been developed and tested. Salvietti and collaborators [31] also demonstrated that it is possible to control a supernumerary robotic finger using EMG signals from frontalis muscles, while Parietti and Asada [32] controlled an extra robotic leg using EMG signals from torso muscles. In most cases, however, kinematic or muscular signals used for controlling additional DoFs have been recorded from body parts not directly involved in the task performed concurrently with the DoFs of the natural limbs. In many real-life conditions, however, such body parts may be involved in the task and thus may not be available to control extra DoFs. Finally, concerning neural null space, a noninvasive brain-machine interface has been used to control a third arm for multitasking [33], but not all participants were able to achieve multitasking.
Here we propose a novel approach to motor augmentation based on the concept of task-intrinsic muscular null space. Musclular null space is the vector space of all muscle activation patterns that do not generate net joint torques. It includes, for example, the co-contraction of two antagonistic muscles, which counterbalance the effect of each other. In many real-life motor tasks, muscular null space is associated to the control of end-effector impedance, especially in presence of unstable interactions with the environment [34 36]. Thus, muscular null space has been successfully used for tele-impedance application, i.e. the control of the impedance of robotic devices through human impedance [37,38]. However, muscular null space can also be used to control extra DoFs. A recent study has demonstrated that muscular null space can be controlled voluntarily to modulate the stiffness of a virtual end-effector during the generation of multidirectional isometric forces [39]. Takagi and collaborators [40] have also recently shown that it is possible to regulate cocontraction of two antagonist muscles to control the vertical position of a 2D cursor while simulaneously controlling the horizontal position with reciprocal activation. More recently, Bräcklein and collaborators [41] have successfully tested an approach in which beta band activity in the neural drive to the tibialis anterior muscle, which does not directly affect the force generated by the muscle, was modulated to control a cursor in a 2D environment. However, no study to date has investigated the possibility of using null space signals extracted from many muscles to directly control extra DoFs while simultaneously performing a task in 3D environment involving multiple DoFs controlled by the same muscles (i.e. using the "task-intrinsic" null space), thus augmenting human motor abilities. This approach differs both from the use of task-extrinsic null space, i.e. from body part not directly involved in the task, and from tele-impedance control.

In this study, we aimed at testing the feasibility of taskintrinsic muscular null space control for motor augmentation by assessing the performance of participants in the simultaneous control of natural and extra DoFs. Moreover, we aimed at assessing whether and how fast null space control ability improves with practice. We designed an experimental protocol in which participants had to displace a cursor in a $3 \mathrm{D}$ virtual environment to reach 8 targets by generating isometric force and simultaneously to control an extra degree of freedom, i.e. the rotation around one axis of the cursor, which had a ellipsoidal shape, through null space activation in arm and/or shoulder muscles. Moreover, participants were instructed to hold the cursor at the target for a given time interval. Thus, our protocol required the simultaneous control of natural and extra DoFs to perform both a spatial and a temporal task.

To quantify subjects' performances and to understand how the task could be optimized to match individual control ability, 
we used a novel index of difficulty (ID), an informationtheoretic metric inspired by Fitts' law. Although Fitts' law general validity has been frequently questioned in the past, Gori and collaborators [42] have proposed an informationtheoretic model of the human motor system for pointing tasks, where the ID is the information about the selection of a target transmitted through a noisy channel. To date, many researches in human motor control have used measured derived from the Fitts' law to evaluate performance in different tasks [43-45]. However, the possibility of considering the time as a "target" itself, i.e. the application of the Fitts' law to temporal control, has been rarely studied [46]. To address this issue, we introduced an ID defined as the sum of a spatial term, related to difficulty in selecting a target by reaching it (i.e. quantifying spatial control ability), and a temporal term, releated to the difficulty in holding the target for a given time interval (i.e. quantifying temporal control ability).

\section{Materials and Methods}

\subsection{Participants}

Eight naïve right-handed participants (mean \pm SD age: 27.5 \pm 7.8 years, age range: $20-45,2$ females) participated in the experiments after giving written informed consent. All procedures have been conducted in accordance with the principles embodied in the Declaration of Helsinki, comply with national regulations, and have been approved by the local ethics committee. All participants had normal or corrected to normal vision and did not report any known neurological disorder or upper right limb injuries.

\subsection{Setup}

The setup used for this work is similar to that used in previous studies $[12,39,47]$. Participants sat on a gaming chair in front of a desktop (Fig. 1A), with the right hand inserted in an orthosis rigidly connected to a 6-axis force transducer (Delta F/T Sensor, ATI Industrial Automation, Apex, NC, USA). Arm and forearm formed a $90^{\circ}$ angle, and the chair was positioned so that the hand was at level of the solar plexus. Car belts immobilized the participant's torso and shoulders. Shutter glasses (GeForce 3D Vision 2, NVIDIA Corporation, Santa Clara, CA, USA), allowed to view stereoscopically a three-dimensional scene displayed on a horizontal mirror, placed over the participant's hand, reflecting the image visualized at $120 \mathrm{~Hz}(60 \mathrm{~Hz}$ for each eye) on a monitor. The scene included a virtual desktop and a cursor (spherical or ellipsoidal) whose position matched the position of the center of the palm when no force was exerted. Real-time feedback of the exerted force was provided as the displacement of the cursor. Cursor motion in three-dimensional space was simulated as an adaptive mass-spring-damper system, subject to the force applied by the participant on the orthosis. The spring constant was set such that the force applied to maintain the cursor stationary at the target was equal to a specific fraction of the magnitude of the participant's maximum voluntary force (MVF, see below). The mass was adjusted adaptively in the range $15-140 \mathrm{~g}$ as a sigmoidal function of the rate of change in the magnitude of the recorded force, to maintain fast responses to changes in force while reducing the effect of noise with stationary force [12].

Surface EMG activity was recorded from fifteen muscles acting on the shoulder and elbow: brachioradialis (BracRad), biceps brachii long heads (BicLong) and short head (BicShort), pectoralis major (PectMaj), anterior deltoid (DeltA), middle deltoid (DeltM), posterior deltoid (DeltP), triceps brachii lateral head (TriLat) and long head (TriLong), infraspinatus (InfraSp), teres major (TerMaj), latissimus dorsi (LatDorsi), lower trapezius (TrapLow), middle trapezius (TrapMid), and upper trapezius (TrapSup). The signal was acquired at $1000 \mathrm{~Hz}$ with active wireless bipolar surface electrodes (Trigno System, Delsys Inc., Natick, MA, USA), bandpass filtered $(20-450 \mathrm{~Hz})$, and amplified with a 1000 gain. Participants' skin, in correspondence to the target muscles, was cleansed with alcohol and electrodes were placed based on recommendations from SENIAM [48] and by palpating muscles to locate the muscle belly and orienting the electrodes along the main direction of the muscle fibers.

Experiment control, data acquisition, and data analysis were performed with custom-written software in MATLAB $\mathbb{R}$ (MathWorks Inc., Natick, MA) and Java ${ }^{\circledR}$.

\subsection{Experimental protocol}

After an initial familiarization with the experimental setup, participants performed 18 blocks with different task conditions. In the first block (MVF estimation), they were instructed to exert their MVF directed towards their chest on the horizontal plane (-y, with y away from the chest along the anteroposterior axis). The maximum of the force recorded in this block was used to normalize target distance in the following blocks.

In the second block (force control, FC), participants were instructed to move, both accurately and quickly, a spherical cursor from the rest position to a target (Fig. 1B), located in one of twenty spatial positions around the rest positions, by applying isometric forces on the orthoses. At the beginning of each trial (rest phase) participants were asked to relax their right arm muscles to maintain the cursor inside a transparent sphere at the centre of the scene, i.e. the rest position, for $1 \mathrm{~s}$. Then, a transparent sphere appeared in one of the twenty target positions (target go event), placed on the vertices of a dodecahedron inscribed into a sphere, centred in the rest position, and whose radius was either $15 \%$ or $25 \%$ the MVF. Participants were asked to reach the target and remain within the target sphere (see Fig. 1A), whose radius exceeded that of the cursor by $2 \%$ the MVF, for $0.5 \mathrm{~s}$ (holding phase). When the cursor was within the target tolerance, the target changed 
color (from gray to yellow). Each target was presented three times, such that each participant performed a total of 120 trials (20 targets $\times 2$ radii $\times 3$ repetitions). The time limit for trial completion was of $4 \mathrm{~s}$. EMG and force data collected from the target go event until the first time the cursor entered the target (dynamic phase) were used to estimate a subject-specific matrix that approximates the mapping of EMG activations onto isometric force (see below EMG-to-force matrix) and its null space. The maximum amplitude of each EMG signal (low-pass filtered with second-order Butterworth; $1 \mathrm{~Hz}$ cutoff) collected during the same phase was used to normalize EMGs during the rest of the experiment. After this block, there was a 5 min pause to process the data.

In the third block (null space modulation, NSM), participants performed a cursor stabilization task that required voluntarily modulation of muscular co-contraction. The EMG data collected in this condition was used to estimate the null space patterns that each participant generated more naturally. Participants had to maintain the cursor inside a target placed at the rest position, whose radius exceeded that of the cursor by $6 \%$ of the MVF, for $1 \mathrm{~s}$ while a simulated sinusoidal force perturbed the motion of the cursor [39]. To reduce this oscillation, participants were instructed to co-contract their right arm and shoulder muscles. The time limit for trial completion was $15 \mathrm{~s}$, with $5 \mathrm{~s}$ of pause between trials. As for the FC block, visual feedback of the cursor being within target tolerance was provided by changing the color of the target. EMG data collected during the holding phase were used to calculate the null space directions to be used for the control the extra degree of freedom (see "Extra Degree of Freedom control" section).

In the fourth block (isometric reaching with ellipsoidal force control, EFC), participants performed an isometric reaching task with an ellipsoidal, rather than spherical as in the FC block, cursor and targets. There were eight targets (3 repetitions), placed on the $x-y$ plane at $20 \%$ of MVF from the origin, with a tolerance of $2 \%$ of MVF, and equally distributed with a $45^{\circ}$ angular distance one from the other $\left(0^{\circ}=+x\right.$ direction, with $\mathrm{x}$ mediolateral axis pointing to the right). This block provided a baseline reference for the following 12 blocks.

In blocks $5^{\text {th }}$ to $16^{\text {th }}$ (null space control, NSC), participants were instructed to both translate and rotate the ellipsoidal cursor (around the intermediate axis of the ellipsoid which was rotated such that it was parallel to the longitudinal axis of the forearm) to match the position and orientation of the target (Fig. 1C). Translation was achieved by exerting force and rotation by generating muscle patterns with a component aligned to specific null space directions, selected using the data collected in the NSM block according to the procedure described below (section "Extra Degree of Freedom control"). Each block was composed of three repetition of trials with nine targets (Fig. 1D) in different $x-y$ positions - the same eight as in the EFC block plus one in the rest position - and with the same orientation corresponding to a $60^{\circ}$ rotation of the ellipsoidal cursor from the rest orientation and a tolerance of $7.2^{\circ}\left(4 \%\right.$ of $\left.180^{\circ}\right)$. The target orientation could be achieved with a null space activation norm of $20 \%$ of the maximum norm recorded during NSM block ("maximum voluntary cocontraction", MVCC). The translation tolerance was also of $4 \%$ of MVF. Participants were instructed to hold the cursor at the target for $1 \mathrm{~s}$. In this case, the target changed color only when the cursor was within spatial and angular tolerances simultaneously. The nine targets were presented in a pseudorandom sequence (cycle). The time limit for trial completion was of $4 \mathrm{~s}$. At the end of each cycle, the score for that cycle was visualized.
A

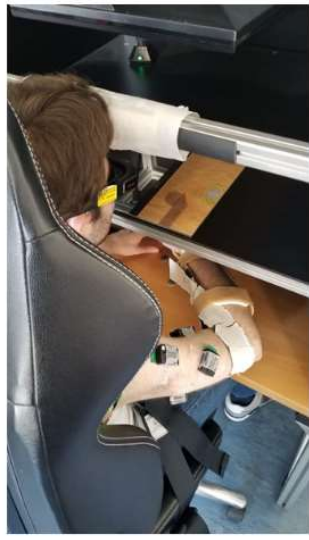

B Force control only

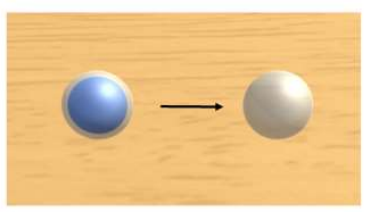

C Simultaneous force control with extra DoF

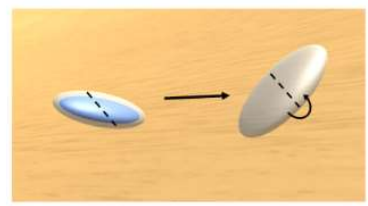

8 targets on the $x-y$ space (+ origin)

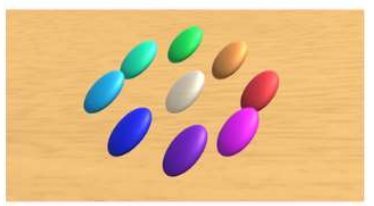

Figure 1. Experimental setup and protocol. (A) Experimental setup: a 3D virtual scene is projected stereoscopically on an horizontal mirror occuding participant's hand, which is attached through an orthoisis at a force fransducer (below the desktop, not visible); wireless sensors are used to collect EMG activity from shoulder and arm muscles. (B) Illustration of the task during force control blocks (the blue cursor moves in the direction of the arrow). (C) Illustration of the task during null space control blocks (the dashed line represent the rotation axis). (D) Target arrangement. (E) Experimental protocol schematic $(\mathrm{MVF}=$ maximum voluntary force, $\mathrm{FC}=$ force control, $\mathrm{NSM}=$ null space modulation, $\mathrm{EFC}=$ ellipsoidal force control, NSC = null space control). 
Finally, the $17^{\text {th }}$ block was a null space control block without visual feedback (hidden NSC) and the $18^{\text {th }}$ block an additional EFC block. A schematic of the experimental protocol is presented in Fig. 1E.

\subsubsection{EMG-to-force mapping}

Isometric generation of submaximal force allowed to use a linear approximation of the relation between the shoulder and elbow muscles activations and the force exterted at the hand:

$$
f=H m,
$$

where $\boldsymbol{f}$ is the tridimensional force vector, $\boldsymbol{m}$ is the 15 dimesional muscle activation vector, and $\boldsymbol{H}$ is the EMG-toforce matrix that maps muscles activations onto force. The matrix $\boldsymbol{H}$ was estimated using multiple linear regressions of each force component, low-pass filtered (second-order Butterworth; $1 \mathrm{~Hz}$ cutoff), with EMG signals recorded during the dynamic phase of the first force control block, low-pass filtered (as the force) and normalized to the maximum EMG activity recorded during the force control block targets at $25 \%$ of the MVF distance. The matrix $\boldsymbol{H}$ was also used to compute the null space matrix $\boldsymbol{N}$, i.e. an orthonormal basis spanning the subspace of vectors $\boldsymbol{n}$ that are mapped by the $H$ matrix onto the null force vector:

$$
0=\boldsymbol{H n}
$$

\subsubsection{Extra Degree of Freedom control}

To characterize the subject-specific directions in the EMG null space to be used for the control of the extra DoF, each participant performed a NSM block. This procedure allowed to identify the directions that each participant could control more naturally and the dimensionality of this subspace. We then selected the directions in the null space with the largest amplitude modulation during the NSM block and used the projection of the instantaneous muscle activity vector onto those directions as the signal to control the extra DoF.

The ideal control variable for null space control should be as small as possible during generation of pure force and have the largest range during voluntary modulation of cocontraction. We investigated three different null space control variables that could be suitable for our task. We tested them on the data previously collected in a task in which participants had to stabilize a cursor by null space activation [39]. Data were collected from eight participants performing five blocks: a force control block (baseline) and four additional blocks. Of these four blocks, the first was a NSM block similar to the one described above, while the other three blocks (force control with perturbation, that here we define "perturbation blocks") required the participant to reach targets using force control and to reduce the oscillation of the cursor induced by a sinusoidal perturbing force by null space activation. In fact, the norm of the null space activation controlled the stiffness of a virtual spring determining the magnitude of the cursor oscillations. The intensity of the sinusoidal perturbation (and therefore of the magnitude of null space activation required to maintain the cursor within the target) increased from the first to the third block.

We tested the following the null space control variables (labelled $f_{D o F}$ ):

- the norm of the projection of the null space activation vector $\boldsymbol{n}=\boldsymbol{N}^{\top} \boldsymbol{m}$, onto the first $n c$ principal components that explain $80 \%$ of data variation in the NSM block after subtraction of the mean vector of null space activation in that block $\overline{\boldsymbol{n}}_{\boldsymbol{c} \boldsymbol{c}}$ :

$$
f_{D o F}=\left\|\boldsymbol{V}_{c c}(:, n c)^{\prime}\left[\boldsymbol{n}-\overline{\boldsymbol{n}}_{c c}\right]\right\|
$$

where $\boldsymbol{V}_{c c}(:, n c)^{\prime}$ represents the transpose of the first $n c$ columns of the matrix of the principal components of the null space activation vectors collected in the NSM block;

- the norm of the projection of the null space activation vector $\boldsymbol{n}$ onto the first $n c$ principal components that explain $80 \%$ of variance of NSM block after subtraction of the mean vector of null space activation in baseline FC block $\overline{\boldsymbol{n}}_{\boldsymbol{b l}}$, taken as a reference of residual, involuntary null space activation:

$$
f_{D o F}=\left\|\boldsymbol{V}_{c c}(:, n c)^{\prime}\left[\boldsymbol{n}-\overline{\boldsymbol{n}}_{\boldsymbol{b l}}\right]\right\|
$$

- the norm of the projection of the null space activation vector $\boldsymbol{n}$ onto the mean null space activation vector in baseline FC block $\overline{\boldsymbol{n}}_{\boldsymbol{b l}}$ :

$$
f_{D o F}=\left\|\overline{\boldsymbol{n}}_{\boldsymbol{b l}}{ }^{\prime} \boldsymbol{n}\right\| .
$$

After calculating all the projection matrices required for computing the three control variables, we assessed the mean value of each variable across the force control block and all the three perturbation blocks for each subject. The different control variables were compared using one-way ANOVA (see "Statistical Analysis" for details). Also the difference between the mean value of each variable across all the three perturbation blocks and the force control mean value was computed to find the method with the largest activation range.

In the NSM block, the perturbation was generated as a sinusoidal force (with different frequencies along different axes) acting on a mass attached to a position (controlled by force) through a spring with an elastic constant that was adjusted in real time according to the norm of the null space activation vector through a logistic function [39]: 


$$
k(n)=\frac{k_{\max }}{e^{-r_{k}\left(\|n\|-\left\|n_{0}\right\|\right)}+1},
$$

Where $\|\boldsymbol{n}\|$ is the norm of the null space activation vector, $k_{\max }=9500 \mathrm{~N} / \mathrm{m}^{2}$ is the spring constant, $r_{k}$ is a variation rate parameter, and $\left\|\boldsymbol{n}_{\mathbf{0}}\right\|$ is the value of the null space norm such that $k(n)=\frac{k_{\max }}{2}$. The value of $\left\|n_{0}\right\|$ was set equal to 2.5 times the minimum norm $\left(\left\|\boldsymbol{n}_{\boldsymbol{m i n}}\right\|\right)$ of the mean null space activation during the static phases of FC block, while $r_{k}$ was calculated using the formula:

$$
r_{k}=-\log \left(\frac{k_{\max } / y_{0}-1}{x_{0}-1}\right)
$$

Where $\left(x_{0}, y_{0}\right)=\left(\left\|\boldsymbol{n}_{\min }\right\|, 500\right)$.

Mean-subtracted holding phase data were used for computing the principal components. We obtained a $\boldsymbol{V}_{\boldsymbol{c}}$ matrix of dimensions $12 \times 12$ whose columns were the principal components of the data. The null space was 12dimensional because the muscle space was 15-dimensional and the task space 3-dimensional. Then, we selected the first $n c$ components that explained $80 \%$ of the total variation of the data (mean value of components \pm SD among participants: 2.1 \pm 0.8 , range $1-3$ ).

Many different functions can be used for mapping the null space control variable onto the control signal for the extra DoF. We chose a logistic function, as the one used in the null space modulation block, because it is positive defined, and participants could then reach the rest position simply by relaxing their muscles. Moreover, it has a smooth and continue derivative, so that there is no need for thresholding, as it would have been necessary for example with a linear function.

Therefore, the control law that mapped null space activation onto cursor rotation angle was defined as:

$$
\theta\left(f_{D O F}\right)=\frac{\theta_{\max }}{e^{-r_{\theta}\left(f_{D o F}-f_{D o F, 0}\right)+1}}
$$

where $\theta$ is the angle of rotation, $\theta_{\max }$ is the maximum angle of rotation, set to $145^{\circ}, r$ is the variation rate, $f_{D o F}$ is the control variable and $f_{D o F, 0}$ is the value of the control value for which $\theta\left(f_{D o F}\right)=\frac{\theta_{\max }}{2}$.

The value of $f_{D O F, 0}$ was computed using $n$ equal to $25 \%$ of the MVCC. The $r_{\theta}$ value was calculated using the formula:

$$
r_{\theta}=-\log \left(\frac{\theta_{\max } / y_{0}-1}{x_{0}-1}\right)
$$

where $\left(x_{0}, y_{0}\right)=\left(f_{\text {DoF }}\left(\boldsymbol{n}_{\min }\right), 0.1^{\circ}\right)$.

\subsection{Data Analysis}

All collected data were visually inspected and trials in which EMG artefacts were detected were discarded. The discarded trials were $13.1 \pm 7.6$ (mean \pm SD over participants) over a total of 536 trials performed by each participant. Trials in the NSC blocks with the target in the central position (i.e. requiring onlu cursor rotation) were not included in the analysis.

\subsubsection{Task performance}

Task performance was evaluated both as the fraction of trial per block in which participants reached the target (reaching success rate), and as the fraction of trial per block in which participants held the cursor in the target for the required time (holding success rate).

Mean holding time and mean angular error per block were also calculated. In each trial, holding time was defined as the longest time interval in which the cursor remained inside the target (maximum value $1 \mathrm{~s}$, the required holding time). The angular error was defined as the mean of the absolute value of the difference of the cursor rotation angle and the target rotation angle over the interval in which the cursor positional error in space was under the threshold of $6 \%$ of MVF.

\subsubsection{Velocity peaks and movement strategies}

Two different velocities of the cursor were calculated: the tangential velocity of the cursor spatial position (therefore related to the force), and the angular velocity of the cursor rotation angle (therefore related to the muscle null space activation).

The two velocities were computed numerically for each trial, after applying a 2nd order Butterworth filter (3 Hz lowpass cutoff frequency) to the cursor position (measured as a fraction of MVF) and to the cursor rotation angle. The movement onset was defined as the first sample after the end of the rest phase (i.e. when the target appeared on the screen) at which the cursor velocity was higher than a threshold equal to three times the mean velocity recorded in the $0.5 \mathrm{~s}$ before the 'target go' event, which is generally equal to zero due to the participant being at rest, but could be greater than zero due to oscillations or noise. The peak velocity was defined as the first maximum after the movement onset.

Movement onset and velocity peaks were analyzed to assess if different participants used different movement strategies. For example, if a participant displaced the cursor first and then rotated it (using muscle null space activation), or vice versa, or if they moved and rotated the cursor simultaneously, or if there was no specific relation between the two movement components.

\subsubsection{Performance analysis}

In addition to success rates, we used information theory to assess individual control ability. We considered the 
information about the instructed target and time interval that is transmitted by each participants when performing a reaching and holding movement. To generalize the assessment of individual ability beyond the performance achieved by each participant with the specific parameters of the experimental protocol (e.g. the target size or the required holding time) we estimated, through a simulation, the information that would have been transmitted with different target sizes and holding times.

The information transmitted accomplishing a reaching task may be quantified by an index of difficulty, as introduced by Fitts [49]. The Fitts' law states that movement time $M T$ in a reaching task is linearly related to an index of difficulty $I D$ :

$$
M T=a \cdot I D+b
$$

The Fitts' ID, for a target of width $W$ and distance $D$ from the origin, in the Shannon-MacKenzie formulation [50], is equal to:

$$
I D=\log _{2}\left(\frac{D}{W}+1\right)
$$

While Fitts' law validity has been questioned because of its unclear theoretical foundations [51,52], Gori and collaborators [42] derived this law with a simple model of the human performance of an aiming task as a communication process. In this model, the source of the message is the target the individual intends to reach ("aiming is choosing"). In the original formulation of Fitts, aiming at a target of width $W$ at distance $D$ is equivalent to selecting one of $n$ linearly arranged targets of width $W$ such that $D=n W$ (Fig. 2A). If the targets can be selected with the same probability, the entropy of the target distribution, i.e. the entropy of the source, is equal to the ID. The message is then sent through a noisy channel, representing the execution of the reaching movement with physiological noise in the neural and the musculoskeletal systems. If the noise results in a distribution of the arrival position with an amplitude less than $W / 2$, aiming at the center of the target allows to always hit the selected target and thus transmitting the message without error. Then, the ID quantifies the information that can be transmitted in an aiming task with negligible error rate, equal to the source entropy for errorless transmission. Apart from its theoretical framework, Fitts' law has been shown to be a robust empirical relation between movement time and the spatial parameters of a task as long as no temporal constraints are set, or if these constraints are relaxed in such a way that they do not influence too much the task itself [53-55].

Since in our task subjects were required to reach the spatial location ( $x y z$ coordinates) of the target and to align the cursor to the target orientation, we can define two distinct indices of difficulty for each one of the two components of the reaching movements (translation and rotation). For the 3D displacement of the cursor position, considering that the tolerance is always the same for the three axis, a displacement ID can be defined as:

$$
I D_{x y z}=\log _{2}\left(\frac{D}{W_{x y z}}+1\right)=\log _{2}\left(\frac{D}{2 R}+1\right) \text {, }
$$

where $D$ is the target distance in \% of MVF, and $R$ is the target radius also in $\%$ of MVF.

Recent research has shown a dependence of the movement time on the target angle for $2 \mathrm{D}$ and $3 \mathrm{D}$ tasks [56,57]. According to our data, the dependence resembles a linear combination of a sine term and a cosine term. Therefore, a better definition of the ID is:

$$
I D_{x y z}=\log _{2}\left(\frac{D}{2 R}+1\right)+c \cdot \sin (\alpha)+d \cdot \cos (\alpha),
$$

where $\alpha$ is the direction angle of the target on the $\mathrm{x}-\mathrm{y}$ plane. The two coefficient $c$ and $d$ were calculated by fitting movement times vs $I D_{x y z}$ in the two EFC control blocks.

For the cursor rotation, a rotation ID can be defined as:

$$
I D_{\theta}=\log _{2}\left(\frac{D}{W_{\theta}}+1\right)=\log _{2}\left(\frac{\theta}{2 \Delta \theta}+1\right),
$$

where $\theta$ is the rotation angle and $\Delta \theta$ is the rotation angle tolerance (Fig. 2B). The total spatial ID can then be defined as the sum of the displacement and rotation indices:

$$
I D_{S}=I D_{x y z}+I D_{\theta}
$$

The application of this ID formulation to our experimental protocol raises three issues. First, Fitts' law has been formulated for an aiming task in which the participant is not required to hold the end-effector at the target location for a specific time interval, but rather to simply hit the target. However, when considering the control an end-effector with myoelectic signals, it may be necessary to provide also a temporal command in addition to a spatial one. Being myoelectric control typically noisier than the natural limb control, it would be then useful to quantify also the target holding performance. Second, the Fitts' law does not consider the actual error rate in the reaching task, assuming that it is low enough to be neglected. This second issue has been addressed by estimating an effective target width for which the error rate is below a given small (but arbitrary) threshold $[45,58,59]$. However, individual ability in aiming at a target can be rigorously quantified using a communication model with transmission errors [42]. Third, to properly assess the individual ability to control the position and orientation of the cursor, we should have used targets of different size and 
different holding time requirements. Indeed, speed-accuracy trade-off functions derived by systematically varying the required accuracy have been used to assess individual skill in manual tasks [60,61]. However, an additional factor in our experimental design would have required a large number of trials making the assessment too long and fatiguing. We therefore opted for an approximate but faster assessment of the dependence of the individual cursor control ability on the specific task parameters by simulating off-line the performance that would have been achieved with different parameters.

Concerning the first issue, we followed the model of a communication system to derive also a temporal ID. Making a parallel with the spatial case, in which we have $n$ targets of width $W$ in a length $D$, we can consider a time interval of duration $T$, which can be divided in $n$ consecutive temporal targets of duration $\Delta t$. In this way, in addition to selecting a spatial target by reaching it, it is possible to select one of the temporal targets by holding at the spatial target until the specific time is elapsed. In addition to considering that "aiming is choosing" (Fig. 2A and B) [42], which means that an individual can choose one target from a set of many by aiming at it, we consider that "waiting is choosing" (Fig. 2C), which means that an individual can choose a "temporal target" from a set of many by waiting for a given time interval before moving. Following this reasoning, an expression for a temporal index of difficulty can be derived as:

$$
I D_{T}=\log _{2}\left(\frac{T}{\Delta t}+1\right)
$$

where $T$ is the duration of the considered time interval, while $\Delta t$ is the duration of the time sub-intervals, defining the required temporal accuracy.
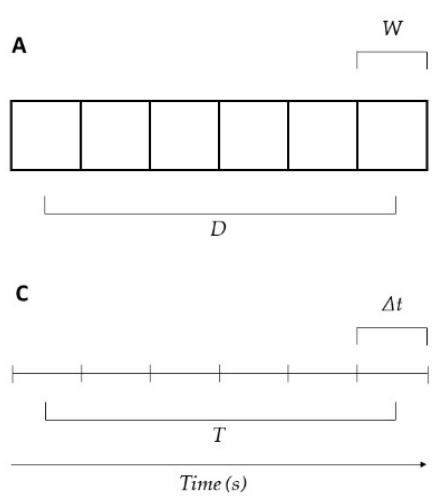

B

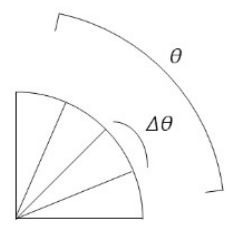

Figure 2. Spatial and temporal ID definitions. Schematic representation of target patterns, according to the Shannon-MacKenzie formulation of the ID, for the three indices: (A) displacement, (B) rotation, and (C) time.
Concerning the second issue, it is possible to calculate the effective size of the target that would satisfy the assumption of negligible error rate (Welford 1968, 1960). Assuming the trial endpoint distribution to be normal, its entropy is equal to $\log _{2} \sigma \sqrt{2 \pi e}$. The quantity $\sqrt{2 \pi e}$ is equal to about 4.133 , which means $\sim \pm 2 \sigma$, that is the $96 \%$ of the area of the distribution. Therefore, if success rate is equal to $96 \%$, the effective width $W_{e}$ of a target and its experimental width coincide. Otherwise, if the standard deviation of the endpoint distribution $\sigma_{e}$ is known, the effective width can be calculated using the formula:

$$
W_{e}=4.133 \sigma_{e}
$$

while if it is not known, the formula using the error rate $\varepsilon$ can be applied:

$$
W_{e}=\left\{\begin{array}{cc}
W \frac{2.066}{Z(1-\varepsilon / 2)} & \text { if } \varepsilon>0.0049 \% \\
W \cdot 0.5089 & \text { otherwise }
\end{array}\right.
$$

where $z$ is the standard score of a distribution with mean $=$ 0 and $\mathrm{SD}=1$.

Nevertheless, this approach has been criticized because it is based on questionable assumptions as the normal distribution of the endpoints and the coincidence that, with $4 \%$ of error rate, the information of a rectangular distribution of width $W_{e}$ is equal to the one of a normal distribution [42].

Therefore, Gori and collaborators have proposed a new index of difficulty that takes into account the error rate. It can be derived using a compound channel with two states (a good state and a bad state), as the Shannon-MacKenzie ID multiplied by the success rate $(1-\varepsilon)$ :

$$
I D(\varepsilon)=(1-\varepsilon) \cdot \log _{2}\left(\frac{D}{W}+1\right)
$$

In our case, we considered the reaching error rate $\varepsilon_{r}$ (or the success rate $\left.\left(1-\varepsilon_{r}\right)\right)$ related to the identification of the target in space, i.e. to the spatial ID, while the holding error rate $\varepsilon_{h}$ (or the success rate $\left(1-\varepsilon_{h}\right)$ ) to the identification of the time interval, i.e. to the temporal ID. Therefore, the corrected ID can be defined as:

$$
I D\left(\varepsilon_{r}, \varepsilon_{s}\right)=\left(1-\varepsilon_{r}\right) \cdot I D_{S}+\left(1-\varepsilon_{h}\right) \cdot I D_{T} .
$$

Concerning the third issue, in our experimental protocol we used only one target size (corresponding to a cursor translation accuracy of $4 \% \mathrm{MVF}$ ), one cursor rotation tolerance (corresponding to $4 \%$ of the MVCC). Moreover, the temporal accuracy required for the holding time (the $\Delta t$ parameter) was not explicitly defined: partipants were required to keep cursor 
in position inside targets for a time $T=1 \mathrm{~s}$. However, since we wanted to assess the individual ability in displacing and orienting the cursor and in holding the target regardless of specific task parameters, we used the data collected in one condition to simulate the performance that participants would have achieved in different conditions. Thus, we computed the reaching performance with targets of different sizes $(6 \%$ to 3 $\%$ of MVF and corresponding \% of MVCC, with a step of $0.5 \%$ ), and the performance for holding the target for the required time with differenent temporal tolerances $(1 \mathrm{~s} \pm 0.1 \mathrm{~s}$ to $1 \mathrm{~s} \pm 0.9 \mathrm{~s}$ with a step of $0.1 \mathrm{~s}$, and $1 \mathrm{~s} \pm 0.999 \mathrm{~s}$, this last being equivalent to just spatial reaching).

As a first step, we estimated the mean movement time (defined as the time interval between the "target go" event and the end of the holding phase) from simulations with different target and holding time tolerances for each participant. Then, we linearly fitted movement times versus total ID to verify that a linear relation still holds when the temporal ID is added.

Finally, an additional measure of performance that can be obtained from the Fitts' law is the throughput, defined as the ratio between the ID and the movement time. The average movement times for each block and target were taken, and the mean across targets was computed. We then estimated the throughput considering only the reaching phase, because the holding phase has a fixed information rate. Whenever a target was not reached in a block, we set the throughput for that target to zero.

\subsubsection{Statistical Analysis}

Statistical analysis was performed using MATLAB. Anderson-Darling test (function adtest) was used to check the normality of the distribution of datasets from the simulation of the different extra DoF control laws. One-way ANOVA test (with multiple comparison with Tukey's honestly significant difference criterion as post-hoc) between datasets composed by the same control methods from all the participants was performed to investigate differences between control methods. Similarly, Kruskal-Wallis one-way ANOVA, after AndersonDarling test, was used to compare reaching and holding success rates, and the $\mathrm{R}^{2}$ of reconstruction of the three force control blocks (one FC and two EFC blocks).

For the NSC blocks, the dependence of reaching and holding success on cycle and target was assessed by fitting a generalized linear mixed model (function fitglme), with the cycle ( 3 cycles per block) and target ( 8 peripheral targets) as fixed effects and participant as random effect. Similarly, the dependence of angular deviation and holding time, on cycle and target was assessed by fitting a linear mixed model (function fitlme). Additionally, a generalized linear model function fitglm) and a linear model (function fitlm) was fitted to the response variables for each participant separately.

Pearson correlation coefficient between force and extra DoF peak velocity times was calculated to assess the correlation between the two velocity peak times across blocks, and Kruskal-Wallis one-way ANOVA, after AndersonDarling test, was used to evaluate differences between the dataset distributions.

\section{Results}

\subsection{Selection of null space control variables}

We performed a simulation on data collected during combined force and muscular null space activity generation [39] to select the variable to be used for the control of an extra DoF. We calculated the mean values for three candidate control variables, which differed for the null space components of the projection of the muscle patterns selected and for the mean muscle pattern used for subtraction. The mean values were calculated during four blocks in which participants generated only force (Force Control, Fig. 3) or force combined with increasing levels of co-contraction (Perturbation Blocks 1-3). After verifying the normality of the distribution of the values of each control variable $(p>0.05$, Anderson-Darling test), we performed a one-way ANOVA on the value of the control variable in each block with the method used to compute the control variable as factor. It gave a pvalue $<0.05$ for all blocks except the one with combined force and co-contraction control with the lowest level of required co-contraction (Perturbation 1 block). Post-hoc comparisons revealed that, for pure force control, the first method, using the projection onto the null space principal components explaining $80 \%$ of NSM block data variance after subtracting the mean NSM block activation vector, presented significantly higher involuntary activation with respect to the other ones ( $p$ $<0.001$ for both comparisons), making it unsuitable for our task. For Perturbation 1 block, there were no significant differences between the methods $(\mathrm{p}=0.33)$. However, with higher levels of co-contraction (Perturbation 2 and 3 blocks) the second method had a higher level of the control variable with respect to the first ( $p=0.03$ and 0.02 , respectively) but not with respect to the third ( $\mathrm{p}=0.29$ and 0.19 , respectively). Nonetheless, the one-way ANOVA performed on the activation differences between perturbation blocks and force control block, with control variable as factor, showed a significant effect of control variable type for all block $(\mathrm{p}<$ 0.001 for all blocks), with post-hoc comparisons revealing higher activation ranges for the second method $(\mathrm{p}<0.05$ for all blocks). According to these results, the second method, using the projection onto the principal components extracted during NSM block after subtracting the baseline mean null space activation vector, was selected as the best choice to control the extra DoF.

\subsection{Force control performance}




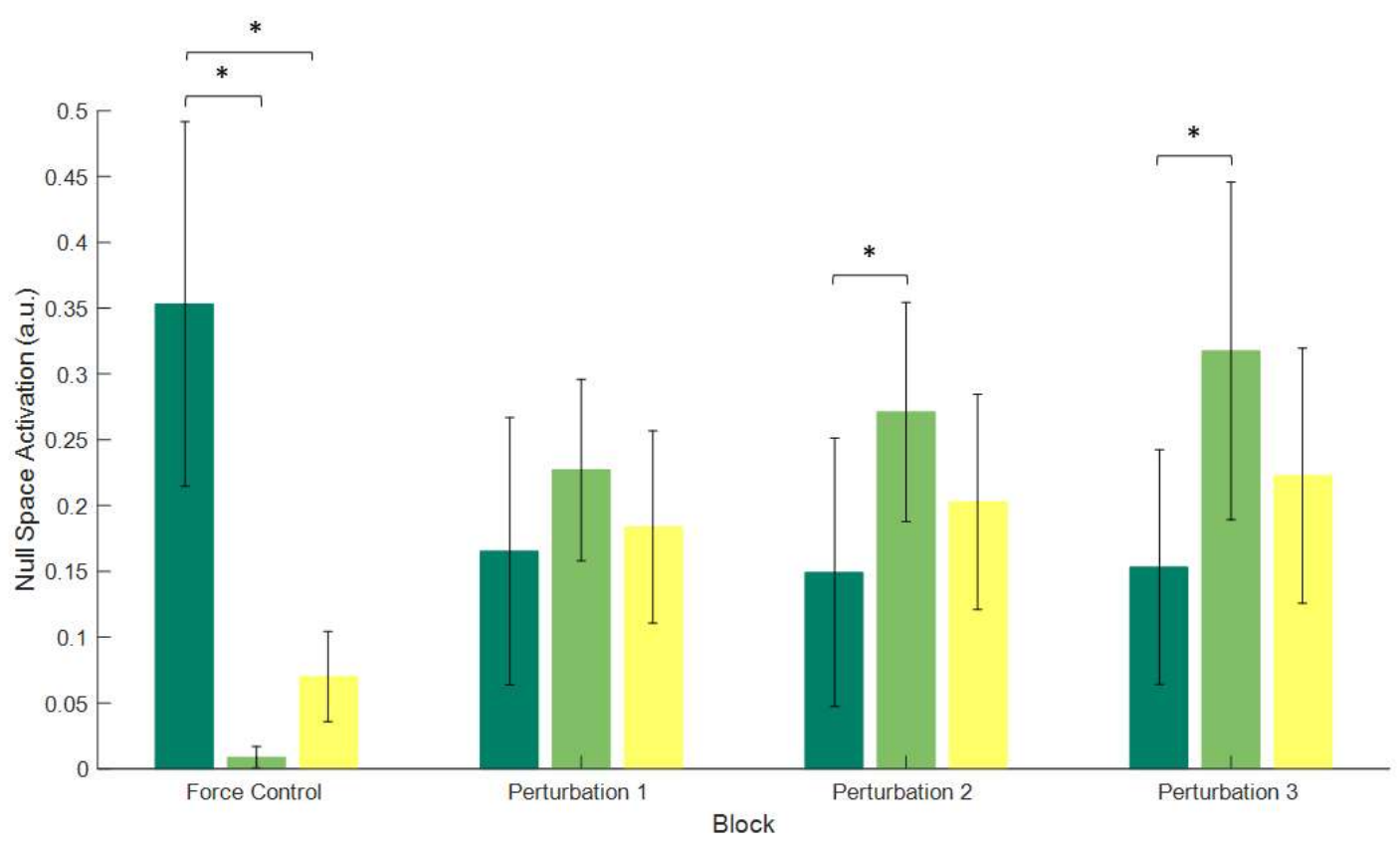

Figure 3. Mean activation of the simulated control variable for three different methods. Bars indicate the mean values of the control variable computed according to the different methods without voluntary co-contraction (Force Control block) and with increasing levels of co-contratcion (Pertubation blocks). Vertical error lines represent standard deviation across participants. Horizontal lines with asterisks indicate significant differences through Tukey's honestly significant difference criterion. The first method (dark green) uses the norm of the projection of the null space activation vector onto the first principal components explaining $80 \%$ of data variation in the NSM block (after subtraction of the mean vector of null space activation in that block). The second method (light green) is based on the norm of the projection of the null space activation vector onto the first set of principal components that explain $80 \%$ of variance of NSM block (after subtraction of the mean vector of null space activation in baseline FC block, taken as a reference of residual, involuntary null space activation), Finally, the third method uses the norm of the projection of the null space activation vector onto the mean null space activation vector in baseline FC block. The second method clearly shows the lower involuntary activation during force control and the widest activation range.

After selecting the method to compute the null space control variable, we recruited eight participants to assess their ability to control simultaneously natural and extra DoF. We first assessed baseline performance in FC. During this block, participants displaced the cursor toward the targets along approximately straight paths, reached the target successfully in $92 \pm 6 \%$ (mean \pm SD across participants) of the trials, and remained in the target for the required time in $63 \pm 19 \%$ of the trials (see Table 1 for individual data). Thus, while reaching the target was easily accomplished by our participants, holding was more challenging. Moreover, holding performance varied considerably across participants, as indicated by its large standard deviation. Similar performances were observed during the initial EFC block, for which the success rates for reaching and holding were, respectively, $88 \pm 25 \%$ and $67 \pm 31 \%$. No significant differences were found between FC and EFC blocks for both reaching and holding performance $(p=0.24$ and 0.56 , respectively, Kruskal-Wallis one-way ANOVA). Therefore, the shape (spherical or ellipsoidal) of cursor and targets did not affect force control performance.

The mean $\mathrm{R}^{2}$ across participants of the three-dimensional force reconstruction during the FC block, was $0.76 \pm 0.11$ (see
Table 1 for individual data). During the initial EFC block, the mean horizontal (rather than three-dimensional, as targets were all planar in this block) force reconstruction $\mathrm{R}^{2}$ was 0.78 \pm 0.13 , and no significant differences were found with respect to the initial FC ( $p=0.46$, Kruskal-Wallis one-way ANOVA). These results support the robustness of the EMG-to-force mapping, which was used for calculating the EMG null space and therefore the variable used to control the extra DoF.

\begin{tabular}{|c|c|c|c|}
\hline Participant & $\begin{array}{l}\text { Reaching } \\
\text { success rate }\end{array}$ & $\begin{array}{l}\text { Holding } \\
\text { success } \\
\text { rate }\end{array}$ & $\begin{array}{l}\text { Force } \\
\text { reconstructio } \\
\text { n R }^{2}\end{array}$ \\
\hline 1 & 0.92 & 0.59 & 0.85 \\
\hline 2 & 0.88 & 0.42 & 0.52 \\
\hline 3 & 0.96 & 0.51 & 0.74 \\
\hline 4 & 0.96 & 0.78 & 0.75 \\
\hline 5 & 0.89 & 0.46 & 0.79 \\
\hline 6 & 0.97 & 0.72 & 0.84 \\
\hline 7 & 0.83 & 0.56 & 0.70 \\
\hline 8 & 0.99 & 0.98 & 0.89 \\
\hline
\end{tabular}

Table 1: individual performance and quality of force reconstruction by EMG-to-force linear mapping for the FC block. 


\subsection{Simultaneous force and null space control performance}

In NSC blocks, participants performed trials with eight ellipsoidal targets, positioned at a distance and with an orientation corresponding to $20 \%$ of MVF and $20 \%$ of MVCC respectively. Additional trials with the target at the rest position and orientation corresponding to $20 \%$ of MVCC, i.e., requiring only cursor rotation, were not included in the analysis.

Differently from FC and EFC blocks, especially in the initial NSC blocks, cursor trajectories were highly variable over repetitions because of the interference between the natural and extra DoFs and the lack of coordination among them. Although participants directed the cursor quite accurately toward the targets, they were less accurate with the cursor rotation (i.e., the extra DoF), which was controlled by null space activation, and the rotation angle often overshoot the target angle and oscillated around it. This is clearly visible in both panels of Fig. 4, where in the first blocks the extra DoF often exceed the upper target threshold (dashed horizontal line). Interference between force and null-space control sometimes also led to an oscillation in the spatial position of the cursor, highlighting the difficulty in simultaneous control of the different DoFs, as it is visible in panel A of Fig. 4. With practice, however, all participants improved in their control of the extra DoF. For example, for all four participants illustrated in Fig. 4 initially (Block 5, darkest lines) the first peak velocity of cursor rotation (vertical lines, middle row) occurred often much later than the peak velocity of the cursor translation (vertical lines, bottom row), but it then occurred progressively earlier with practice (Blocks 8-16, lighter lines).

Mean success rate across participants in target reaching and holding increased during the 12 NSC blocks. Reaching success rate progressed from $72 \pm 26 \%$ in the first block to 93 $\pm 11 \%$ in the last block. Holding success rate was initially

A

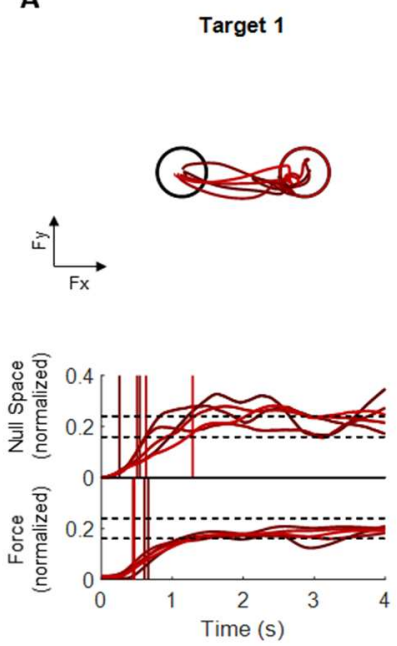

Target 3

Target 5

B

Participant 2
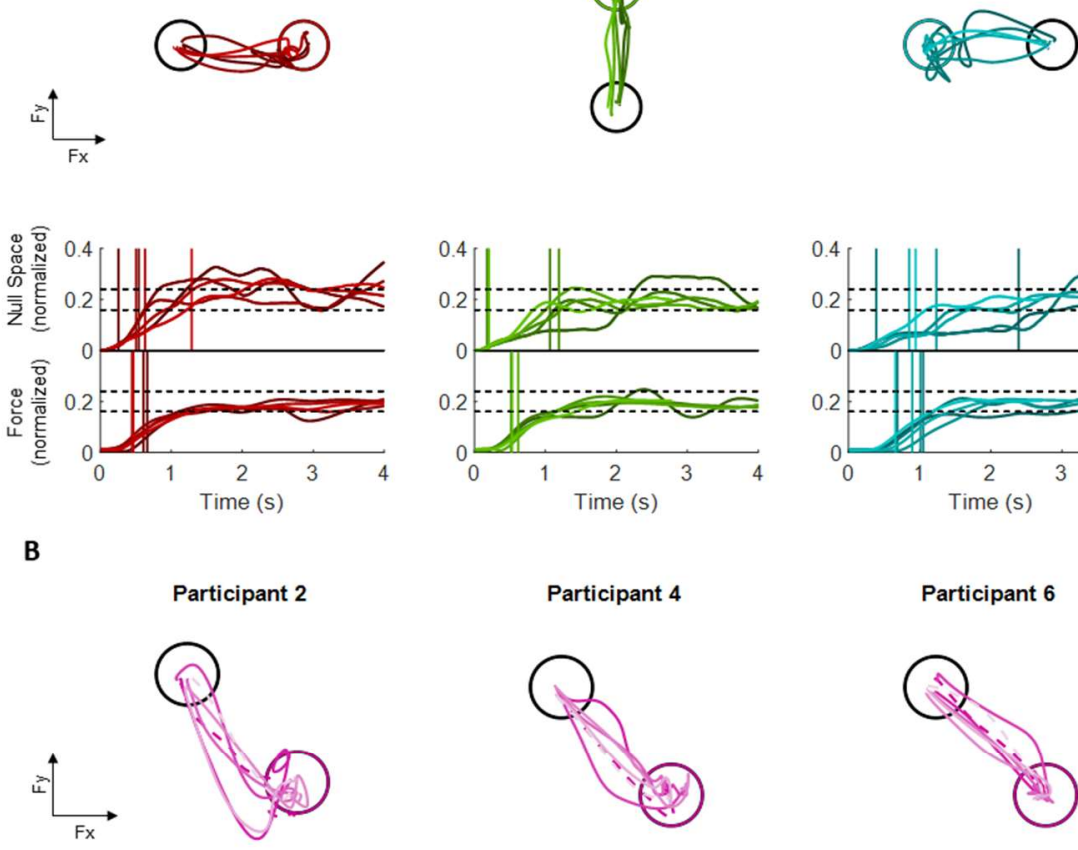

Participant 4
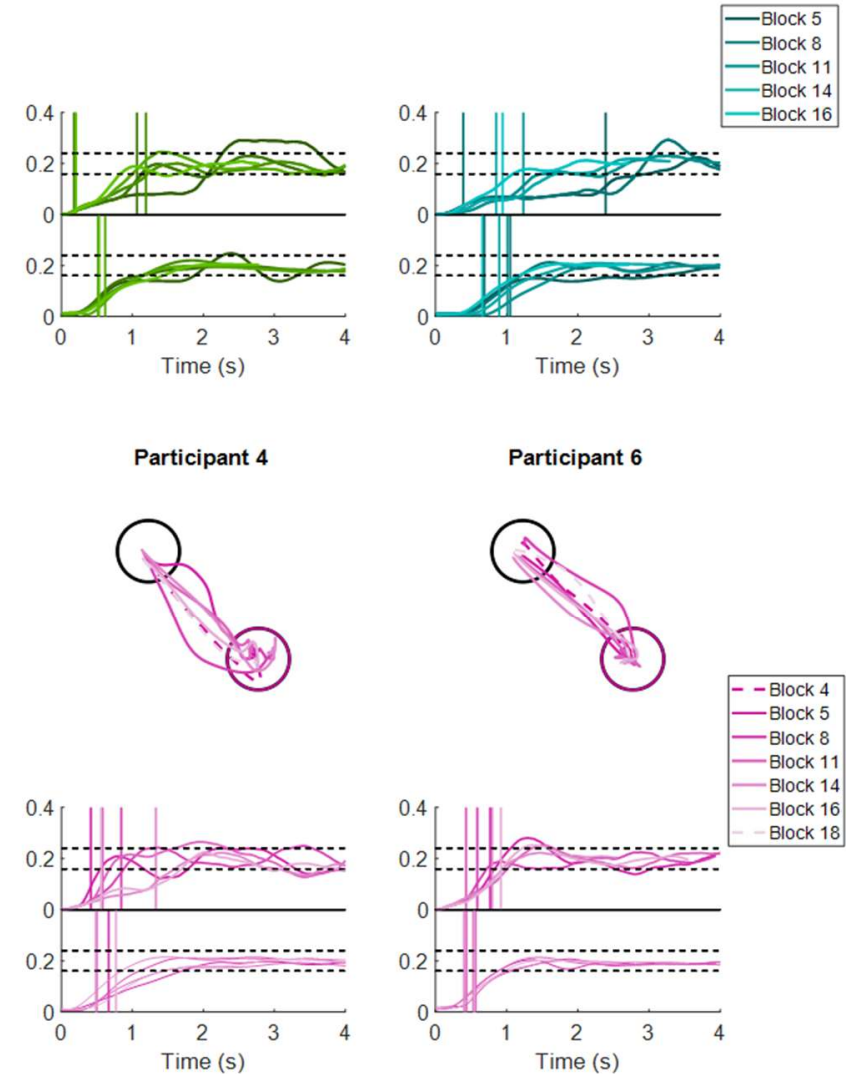

Participant 6

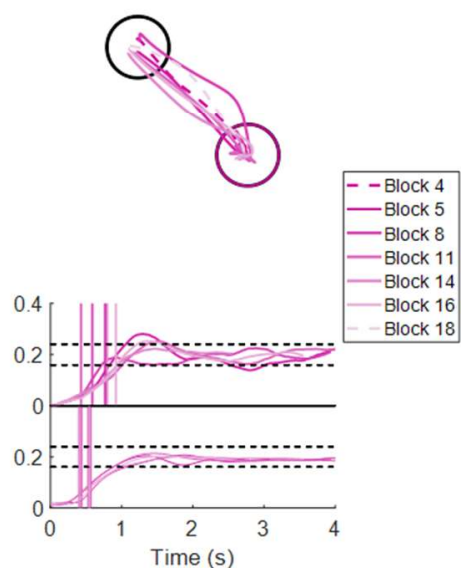

Figure 4. Examples of task performance during NSC blocks. (A) Example of cursor trajectories to different targets (1, 3 and 5) for participant 6: for each target (column) the plot on top shows the trajectory in x-y plane, the middle plot shows the evolution in time of the normalized null space control variable, and the plot on bottom the normalized force. To illustrate the temporal evolution of trajectories the saturation of the colors decreases with block number: trajectories became straighter over blocks. Vertical lines show the time of first velocity peak. (B) Example of trajectories in the x-y plane (top row) and time evolution of null space control variable (middle row) and force (bottom row) to one target (8), for participants 2, 4, and 6 (columns). Trajectories in the $\mathrm{x}-\mathrm{y}$ plane during EFC are also shown for comparison (dashed lines, top row). 
low, $12 \pm 12 \%$ in the first block, and achieved a maximum value of $43 \pm 31 \%$ (Fig. $5 \mathrm{~A}$ and $\mathrm{B}$ ). The mean movement time across participants decreased over blocks, with a starting value of $2.79 \pm 0.54 \mathrm{~s}$ and an ending value of $2.02 \pm 0.54 \mathrm{~s}$. The mean holding time across participants increased, achieving the highest mean value of $0.70 \pm 0.29 \mathrm{~s}$, while the mean angular error decreased below the required target threshold of $7.2^{\circ}$ $\left(6.69 \pm 2.18^{\circ}\right.$ for the last block, minimum mean value achieved) (Fig. 5C and D).

A generalized linear mixed model analysis, with cycle (i.e., a subdivision of a block, with three cycles per block) and target as fixed effects and participant as random effect, showed a significant dependence of both reaching and holding success rate on cycle $(\mathrm{p}<0.001$ for both variables, with a slope of 0.047 and 0.041 , respectively), indicating a significant increase in average performance with practice. The effect of target was also significant for both reaching and holding ( $\mathrm{p}=$ 0.001 and 0.041 , respectively), which means performances were not equal across targets. In fact, targets 4,5 and 8 showed lower mean reaching success rate with respect to target 1 , taken as reference $(p=0.006,0.002$ and 0.004 respectively), while target 6 presented higher holding success rate $(\mathrm{p}=$ 0.022).

Remarkably, there was substantial inter-individual variability in performance, especially for target holding, as indicated by the large standard deviation (Fig. 5A, B, C and D). For this reason, we also analyzed the data of individual participants separately, fitting them with a subject-specific generalized linear model with cycle and target as fixed effects. Individual performance curves are plotted in Fig. 5E and 5F. For reaching success rate, we found a significant effect of cycle only in participants 2, 3 and 6 (Table 2). This is because
A

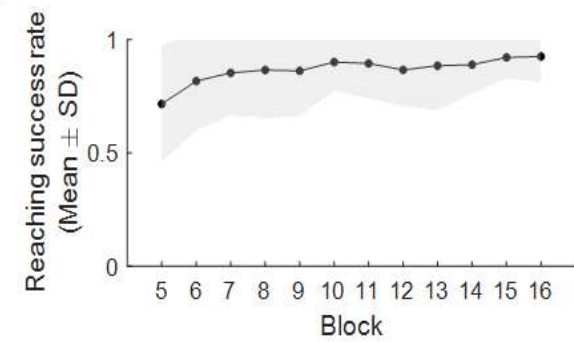

B

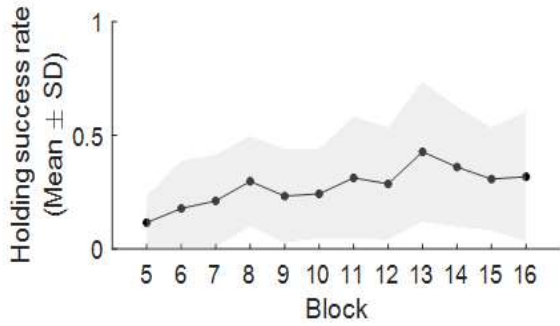

C

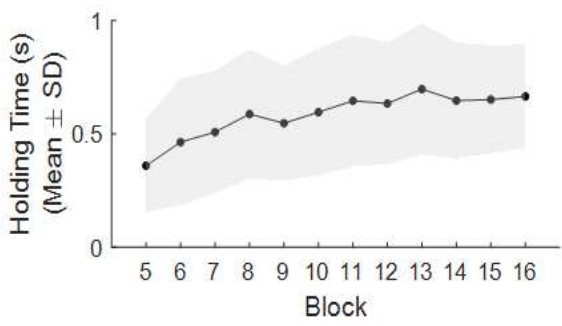

D

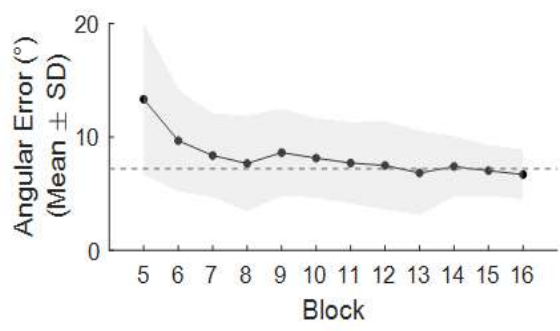

E

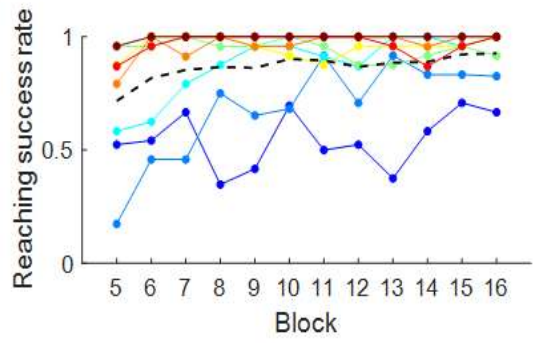

$\mathbf{F}$

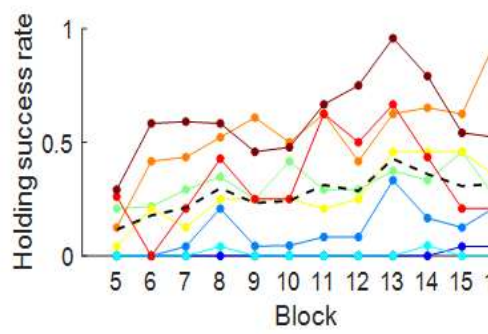

G

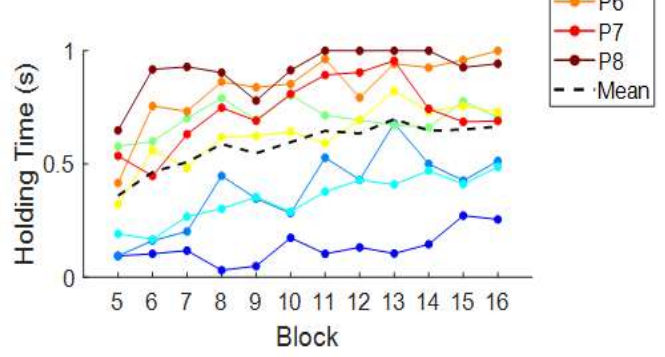

H

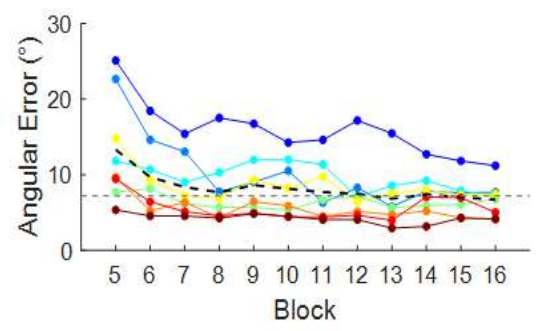

Figure 5. Simultaneous force and null space control performance. Reaching success rate, holding success rate, holding time and angular error (left column, mean across participants; right column, data from all participants). Shaded areas represent mean $\pm \mathrm{SD}$, and the gray dashed line in panel $(\mathrm{H})$ represents target tolerance for angular error. 
all the other participants, except participant 1, had high reaching success rate from the beginning of the experiment. Participant 1 was instead rather erratic, with a large variability in success rate from block to block, and always below $80 \%$. For holding success rate, we found a significant effect of cycle for participants 2, 5, 6, 7 and 8 (Table 2). A significant target effect on reaching performance was found for participants 1 , 2, 3 and 4 and on holding performance for participants 4, 5, 6, 7 and 8 (Table 3). These results indicate that, with practice, some participants improved their control skills in reaching or in holding while others did not, and that such skills were not equal across the different directions.

Linear mixed models, with cycle and target as fixed effects and subjects as random effect, showed a significant dependence of holding time and angular error on cycle $(\mathrm{p}<$ 0.001 for both variables, slope 0.009 and -0.12 , respectively). Target effect was not significant for holding time $(\mathrm{p}=0.09)$, while it was significant for angular error $(p<0.001)$.

Holding time and angular error individual curves are plotted in Fig. 5G and 5H. Linear models fitted separately to each individual participant showed a significant cycle effect on holding time for all participants except participant 4, while for the angular error a significant cycle effect was found for all participants except participant 4 and 7 (Table 2). Both these participants had values close to their best values since the beginning of the experiment. Target effect on holding time was significant for all participants (while it was not the case when considering them together) and on angular error for all participants except participants 6 and 7 (Table 3). In sum, this analysis highlighted that, even when success rate does not increase significantly, improvements can be observed in continuous parameters such as holding time and angular error.

\begin{tabular}{|c|c|c|c|c|}
\hline Subject & $\begin{array}{c}\text { Reaching } \\
\text { success } \\
\text { rate }\end{array}$ & $\begin{array}{c}\text { Holding } \\
\text { success } \\
\text { rate }\end{array}$ & $\begin{array}{c}\text { Holding } \\
\text { time }\end{array}$ & $\begin{array}{c}\text { Angular } \\
\text { error }\end{array}$ \\
\hline 1 & 0.26 & 0.17 & $<0.001^{*}$ & $<0.001^{*}$ \\
\hline 2 & $<0.001^{*}$ & $0.002^{*}$ & $<0.001^{*}$ & $<0.001^{*}$ \\
\hline 3 & $<0.001^{*}$ & 0.94 & $<0.001^{*}$ & $<0.001^{*}$ \\
\hline 4 & 0.14 & 0.17 & 0.20 & 0.10 \\
\hline 5 & 0.65 & $<0.001^{*}$ & $<0.001^{*}$ & $<0.001^{*}$ \\
\hline 6 & $0.005^{*}$ & $<0.001^{*}$ & $<0.001^{*}$ & $<0.001^{*}$ \\
\hline 7 & 0.61 & $0.02^{*}$ & $<0.001^{*}$ & 0.09 \\
\hline 8 & 0.33 & $0.003^{*}$ & $<0.001^{*}$ & $<0.001^{*}$ \\
\hline
\end{tabular}

Table 2: p-values for the effect of cycle on success rate (reaching and holding), holding time and angular error. The asterisk indicates $\mathrm{p}<0.05$.

\begin{tabular}{|c|c|c|c|c|}
\hline Subject & $\begin{array}{c}\text { Reaching } \\
\text { success } \\
\text { rate }\end{array}$ & $\begin{array}{c}\text { Holding } \\
\text { success } \\
\text { rate }\end{array}$ & $\begin{array}{c}\text { Holding } \\
\text { time }\end{array}$ & $\begin{array}{c}\text { Angular } \\
\text { error }\end{array}$ \\
\hline 1 & $<0.001^{*}$ & 1 & $0.048^{*}$ & $<0.001^{*}$ \\
\hline 2 & $<0.001^{*}$ & 0.574 & $<0.001^{*}$ & $<0.001^{*}$ \\
\hline 3 & $0.013^{*}$ & 1 & $0.015^{*}$ & $0.029^{*}$ \\
\hline
\end{tabular}

\begin{tabular}{|c|c|c|c|c|}
\hline 4 & $0.029^{*}$ & $0.007^{*}$ & $<0.001^{*}$ & $<0.001^{*}$ \\
\hline 5 & 0.287 & $<0.001^{*}$ & $<0.001^{*}$ & $<0.001^{*}$ \\
\hline 6 & 0.999 & $<0.001^{*}$ & $0.001^{*}$ & 0.072 \\
\hline 7 & 0.726 & $0.005^{*}$ & $0.004^{*}$ & 0.379 \\
\hline 8 & 1 & $<0.001^{*}$ & $<0.001^{*}$ & $<0.001^{*}$ \\
\hline
\end{tabular}

Table 3: p-values for the effect of target on successful trials fraction (reaching and holding), holding time and angular error. The asterisk indicates $\mathrm{p}<0.05$.

We then investigated the force control and the null space control performances separately, i.e., the success rate for reaching and holding considering only either the position or the rotation of the cursor (Fig. 6). The separate performances were better than the combined performance, which was provided as feedback to the participants during the experiment (as the change of color of the target when both position and orientation of the cursor were within the target tolerance). All participants achieved a $100 \%$ reaching success rate in at least one block for both force control and null space control separately (maximum mean \pm SD across participants: $99.5 \pm$ $1.4 \%$ and $98.9 \pm 1.8 \%$, respectively). Holding success rate raised to $89 \pm 12 \%$ for force control, with 4 participants achieving $100 \%$, and $60 \pm 32 \%$ for null space control. It was therefore the lack of coordination in displacing and rotating the cursor that significantly affected the global performance.

A generalized linear mixed model analysis highlighted a significant dependence on cycle for both reaching and holding of both force and null space control performance $(p<0.001$ for all cases, slope of $0.09,0.05,0.05$ and 0.04 for force reaching, force holding, null space reaching and null space holding respectively). Target effect was significant only for null space reaching $(\mathrm{p}<0.001)$, while it was not for force reaching $(\mathrm{p}=0.13)$, force holding $(\mathrm{p}=0.15)$ and null space holding $(\mathrm{p}=0.58)$.

The analysis of the performances of each participant separately revealed different individual strategies, which were not evident when considering success rate for combined force and null space control. For example, participant 1 showed a significant cycle effect in all success rates except null space reaching, showing an improvement not visible with simultaneous control success rate. This participant, together with participant 2 , was the only one with a significant improvement in force reaching, while in force holding participants 3, 6 and 7 also showed a significant improvement together with 1 and 2 . In null space reaching only participants 2 and 3 had a significant cycle effect; nonetheless, all participants except participant 4 had significant cycle effect in null space holding. It is worth noting that participant 4 increased their performance in null space holding, but after nine blocks, performance started to decrease probably due to fatigue and/or distraction.

In the final EFC block, after the NFC blocks, mean success rates across participants for reaching and holding were respectively $95 \pm 12$ and $77 \pm 24 \%$. No significant differences 

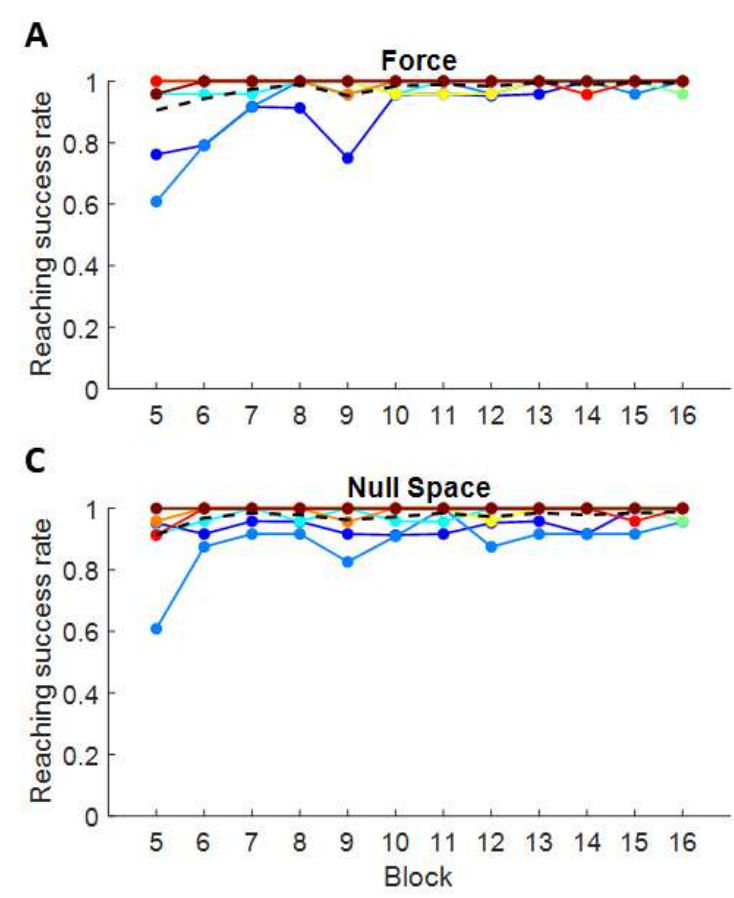

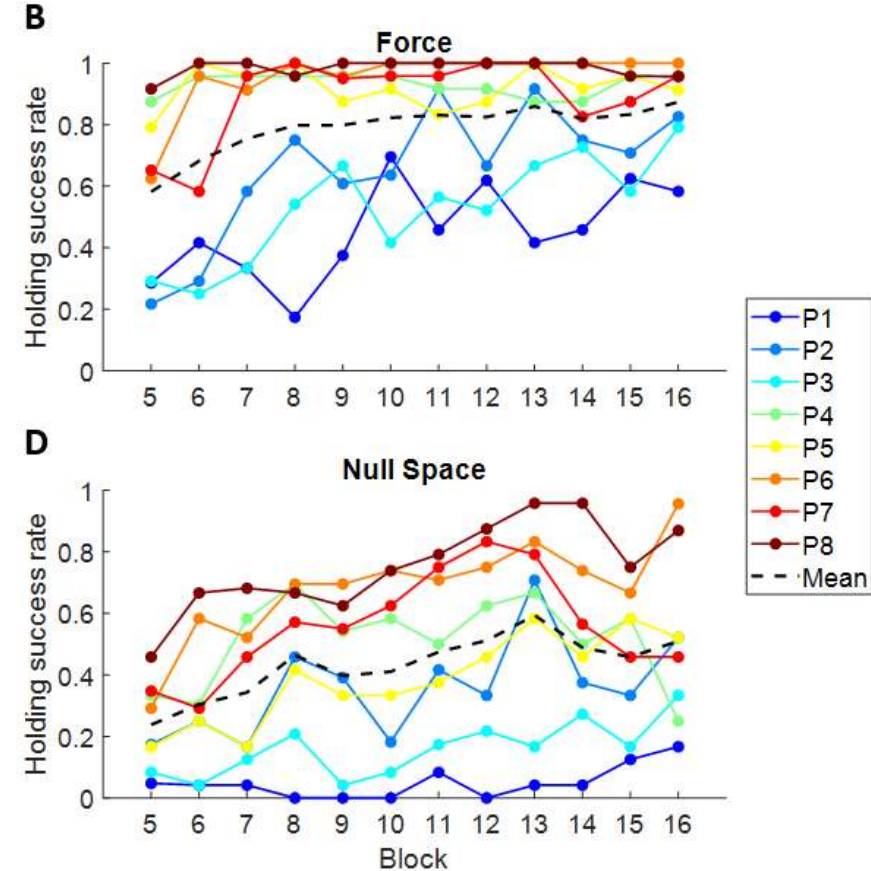

Figure 6. Separate force control and null space control performance. Reaching and holding success rates for force control (first row) and null space control (second row) are shown separately. The black dashed lines represent mean across participants.

were found between initial and final EFC blocks, for both reaching and holding $(\mathrm{p}=0.19$ and 0.48 , respectively, Kruskal-Wallis one-way ANOVA). This indicates that practicing simultaneous force and null space control did not affect force control alone. The mean horizontal force reconstruction $\mathrm{R}^{2}$ across participants during the final EFC block was $0.67 \pm 0.23$, and no significant differences were found with respect to the initial EFC block $(\mathrm{p}=0.48$, KruskalWallis one-way ANOVA). This suggests that null space control did not affect standard force control patterns even after prolonged practice.

\subsection{Peak velocity times and movement strategies}

We analyzed peak velocities to better characterize the different strategies of individual participants. Each participant showed a specific timing of the peak velocity for cursor displacement and for cursor rotation. Some participants first rotated the cursor and then displaced it, others first displaced the cursor and then rotated it, and others performed both movements simultaneously. Furthermore, peak velocity times were not constant over blocks, and they decreased or increased depending on the participant and on the specific target. As can be seen in Fig. 7, the high SD values of the peak velocity times in individual blocks indicate a large variability across targets.

Kruskal-Wallis one-way ANOVA, with peak type as factor, was performed to compare translation and rotation peak velocity times of each participant. This revealed a significant difference between the translation and rotation peak velocity times for all participants $(p=0.002$ for participant $4, \mathrm{p}<0.001$ for participants $1,3,5,7$, and 8) except 2 and $6(\mathrm{p}=0.82$ and 0.42 respectively), although participant 6 had a high variability in rotation peak velocity times across targets. It is also worth noting that, among the participants with significant differences between the two times, only participant 4 had significantly earlier rotation velocity peak than displacement velocity peak.

Translation and rotation peak velocity times showed a strong positive correlation across blocks for participants 2 and 5 (Pearson correlation coefficient $r=0.87$ and 0.83 , respectively) considering all targets directions together, with both mean times decreasing over time (blocks). Moderate positive correlation was found for participants 1 and $6(r=$ 0.68 and 0.52 , respectively), with both mean times also decreasing over time. Moderate negative correlation was instead found for participant $3(r=-0.42)$, with both mean times decreasing up to block 10 , after which the rotation peak time increased. Weak negative correlation was found for participant $4(r=-0.23)$, while no significant correlation was found for participant $8(r=0.09)$, with a constant displacement peak velocity time and a decreasing rotation peak velocity time.

\subsection{Individual null space control ability}

Because of the high variability among the participants in the performance metrics that we analyzed, such as success rate and holding time, which depend on task conditions such as target and time tolerances, we wondered if it was possible to generalize the assessment of individual ability in the 

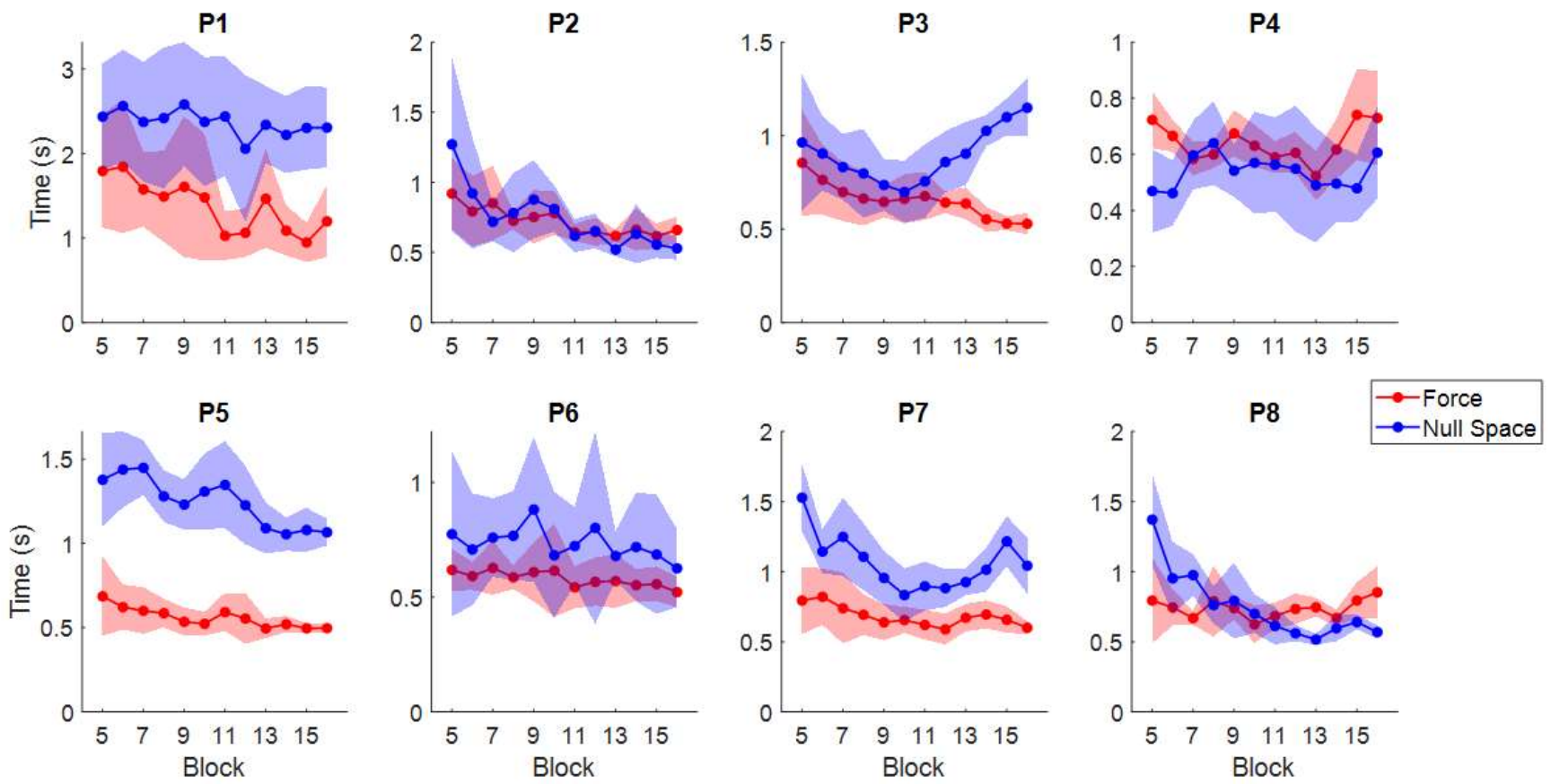

Figure 7. Individual movement strategies. Mean peak velocity times across targets, over null space control blocks, for both force and null space control variables. Shaded area represents standard deviation.

simultaneous control of natural and extra DoF. To this aim, we used an information theory approach inspired by Fitts' law, with an ID comprising a spatial term for the reaching phase and a temporal term for the holding phase. We estimated the performance with different target sizes and holding time tolerance with a simulation.

For the reaching phase only, due to the variability in movement time for the different directions, the linear fit of the movement time itself as a function of the Shannon-MacKenzie ID resulted in a $\mathrm{R}^{2}$ of $0.26 \pm 0.17$ (mean $\pm \mathrm{SD}$ among participants). The linear fit was significant for seven participants $(\mathrm{p}<0.001$ for participants 2 to 8 , while $\mathrm{p}=0.40$ for participant 1), which supports the validity of the Fitts' model for reaching. The plot of the corrected spatial ID as a function of the target size (Fig. 8A) shows that the smallest target is not always the one that allows maximizing the transmitted information. While simulated performances appear to be similar for what concerns the largest possible target, decreasing target size does not always lead to an increase in the transmitted information, because the increase in the total available information associated with smaller targets is overcome by a decrease in success rate. This means that a specific target size can maximize information transmitted through reaching, and it is strictly dependent on participants' ability.

On average, the throughput, calculated as the ID divided by the movement time for reaching, increased during NSC blocks (Fig. 8B). This is expected, as the movement time for reaching also decreased among blocks. This result indicates that with practice participants moved faster while keeping good accuracy.

When considering both the reaching and the holding phase, the linear fit of the simulated movement time as a function of combined ID resulted in a mean $\mathrm{R}^{2}$ of $0.56 \pm 0.18$, and all fits were significant $(\mathrm{p}<0.001$ for all participants). This means that a linear relation still holds when the ID also includes a temporal term.

Introducing the additional temporal ID generally affects the target size at which a participant can transmit the maximum information, as can be seen in the example of participant 8 illustrated in Fig. 8C. While for the reaching ID the best target size was $3.5 \%$ of MVF/MVCC, for holding the best target size was $4 \%$, with a time tolerance of $0.1 \mathrm{~s}$. Moreover, not all participants had their maximum information transmitted for the same time tolerance, indicating that also this quantity depends on participants' ability in holding the cursor in a fixed position.

Finally, the maximum information transmitted when reaching and holding (i.e., the maximum value of information among all the simulated conditions, Fig. 8D) also increased with practice. This means that participants improved with practice their ability to control concurrently natural and extra DoFs, both spatially and temporally.

\section{Discussion}

The control of an extra limb or end-effector while simultaneously performing movements with the natural limbs 
A

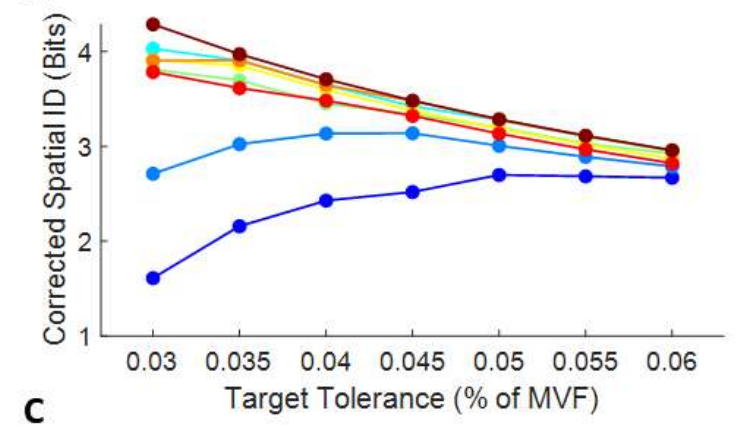

C

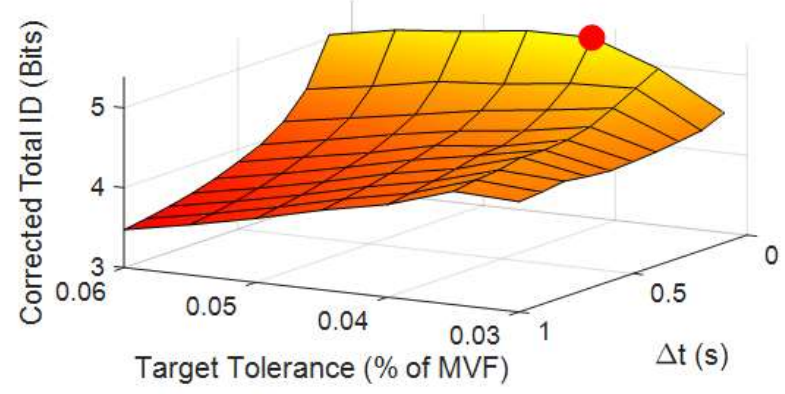

B

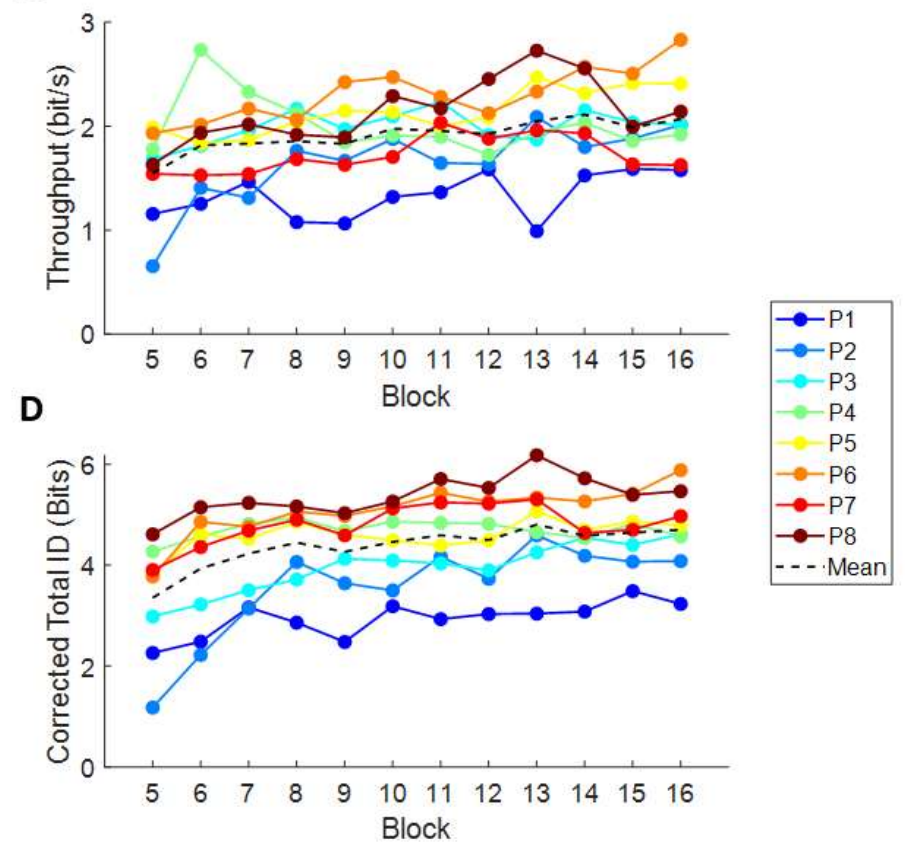

Figure 8. Assessment of individual control ability. (A) The corrected spatial ID (mean among the last three NSC blocks, estimated through a simulation) is shown as a function of target tolerance, showing the optimal target size for reaching of each participant. (B) Throughput as a function of block number. The dashed black line corresponds to the mean value across blocks. (C) Example of corrected total ID (mean among the last three NSC blocks) as a function of target and time tolerances for participant 6 . The red dot indicates the maximum value of transmitted information achieved by the participant among all the simulated conditions; the temporal evolution of its value is reported in orange curve of panel (D). It is worth noting that other maxima could be present outside the space covered by the simulation, and that the curve for $\Delta s=0.999$ (reaching condition) is equivalent to the one present in the panel (A). (D) Time evolution of the maximum information transmitted (among all the simulated conditions, i.e. the maximum of the surfaces such as that shown in panel (C)) for each block and participant. The dashed black line corresponds to the mean value across blocks.

requires using signals that do not interfere with limb motion $[26,62]$. As a first step towards the ambitious goal of augmenting human motor capabilities, we tested whether simultaneous control of natural and extra DoFs through isometric force and intrinsic muscular null space signals is feasible. We developed a control interface in a virtual environment using isometric force generated at the hand to control the translation of an ellipsoidal cursor and, concurrently, muscle-to-force null space activations, i.e., patterns of muscular activations that do not generate force, to control the rotation of the cursor around one axis. We assessed how well 8 participants controlled the end-effector with such interface in a reaching task that required translating and rotating the cursor to match the position and orientation of 8 ellipsoidal targets, thus testing spatial control, and maintaining the cursor in the target for a $1 \mathrm{~s}$, thus also testing temporal control. The results indicate that such an application of muscular null space is feasible, since after a moderate amount of practice average reaching performance was close to $100 \%$. Furthermore, all the participants showed improvements in different performance parameters with practice, such as an increase in reaching and holding success rate, a reduction of angular error, and an increase of holding time. However, we found remarkable inter-individual differences in task performance, learning capabilities, and strategies to coordinate natural and extra DoFs.

There are three kinds of null spaces that can be defined for the human motor system: kinematic, muscular, and neural [26]. Moreover, when considering null space signals to be used for controlling extra DoF during the performance of a task, we can define as task-extrinsic those null space signals generated by body parts, muscles, or neural circuits not directly involved in the task, and task-intrinsic those signals directly involved. Here we considered task-intrinsic muscular null space signals for extra DoFs control. Muscular null space may be a convenient choice for augmentation since it represents a trade-off between the desirable (low noise and non-invasiveness) and potentially limiting (low dimensionality) characteristics of the kinematic null space and the desirable (high dimensionality) and limiting (invasiveness) characteristics of the neural null space. A larger dimensionality of the null space is desirable because it allows for more flexibility in the selection of the dimensions to be used for control. Additionally, using intrinsic muscular task null space may avoid interfering with the performance of additional tasks involving other body parts. For example, 
using null space signal from arm muscles to control extra DoFs participating to the main task performed by the arms (e.g., an extra robotic limb positioning an object being manipulated by hands) may allow to perform secondary tasks such as standing or walking.

Our approach is novel because it is the first time that an intrinsic muscular null space signal extracted from multiple arm muscles is used to control an extra DoF. Many applications of extrinsic kinematic null space have been proposed in the past, such as end-effectors controlled by foot motion [27,63-65], and a third thumb controlled through the big toe [28]. Extrinsic muscular null space has been used for the control of a sixth finger using facial muscles [66] or for the control of external devices using torso muscles [67,68]. Others studies have focused instead on the intrinsic kinematic null space, such as a third limb controlled through force sensors on the fingertips [69]. Applications of neural null space for the control of external devices through brain-machine interfaces, such as a third arm [33] or one of the two DoFs of a cursor in a 2D virtual environment [70,71], have also been reported. However, the possibility of using intrinsic muscular null space for augmentation has received less attention. A recent study has shown that activity in the beta-band of spiking activity of motor neurons identified from high-density EMGs electrode from a single muscle could be suitable to control additional DoFs concurrently with natural limb motion [41], but possible interference with other muscles was not directly monitored. Another recent study [40] has demonstrated the possibility of controlling the vertical displacement of a cursor in a $2 \mathrm{D}$ environment through co-contraction of two antagonistic muscles (pectoralis major and posterior deltoid) while controlling the horizontal displacement through the reciprocal activity of the two muscles. With respect to these recent studies, our interface allowed to directly test the feasibility of simultaneous control in a scenario closer to real-life, i.e., in a $3 \mathrm{D}$ virtual environment, of 3 natural DoFs (cursor translation) and an extra DoF (cursor rotation), for a total of 4 DoFs controlled simultaneously. Moreover, our task-intrinsic muscular null space signal was extracted from many muscles involved in the reaching/holding task. We could assess the interference between the different DoFs and the relative muscle activations, showing that participants could learn to reduce such interference with practice. In principle, our approach could also be extended to the control of multiple extra DoFs by selecting different components in the intrinsic muscular null space. However, further investigation is needed to assess how performance and learning rate depends on the number of extra DoFs.

Because of the high inter-individual variability among our participants, we developed an assessment framework based on information theory inspired by Fitts' law to assess individual control ability independently from the performance observed with specific task parameters. In fact, performance metrics such as success rates are strictly dependent on the specific task conditions used in the assessment. In contrast, evaluating performances in terms of an ID, such as the one proposed in the Fitts' law, allows to generalize an individual's performance and extrapolate it from the specific context, representing them as transmitted information and giving a measure of the effective spatial accuracy limits of a participant. Tasks with larger and closer targets can be easily accomplished with high success rates but low spatial accuracy, which means low information transmitted, i.e., the possibility of choosing a smaller number of targets in a given task. On the other hand, tasks with smaller and farther targets are more difficult, requiring high spatial accuracy which is equivalent to more spatial information transmitted. Since its original formulation [49], Fitts' law has been widely employed to evaluate human performance during tracking [72], myoelectric control [73], prostheses control [74], and humancomputer interaction (HCI) $[45,50,75]$. Fitts' law captures the speed-accuracy tradeoff typically observed in human aimed movements by relating movement duration to an ID defined according to target distance and size. Thus, since the ability to accurately control an end effector depends on the speed of movement, motor control ability should be assessed according to a speed-accuracy trade-off function rather than by accuracy alone [60]. However, the ID itself, corrected through the success rate of a participant, as a measure of average transmitted information [42], can be taken as a metric for performance evaluation related to spatial accuracy and control ability.

While various formulation of Fitts' law have been developed to adapt to different tasks or to correct the ID to account for target misses $[42,58,76]$, Fitts' law has always been considered in the spatial domain, with time taken into account only in the form of temporal constraints influencing task execution $[53,54]$. However, it may be sometimes necessary to evaluate the performance also in temporal domain, such as to evaluate whether an individual is able to perform an action at the right time or for the required time lapse. To account for the effects of temporal targeting, i.e., a task in which spatial distance is minimal and for this reason movement time can be considered constant and close to zero, a recent study applied Fitts' law to a temporal pointing task, in which the user must only decide when to perform an action (in this case, pressing a button when the cursor is inside targets) [46]. Assuming a Gaussian response distribution for the endpoints, the error rate could be expressed as a function of an ID equal to the logarithm of a temporal target distance divided by a temporal target width. Similarly, to take into account the holding phase in our task, which can be considered a temporal task, we hypothesize that the total information transmitted performing the task is equal to the sum of two IDs, a spatial ID resembling the classical Fitts' index, and a temporal ID similar to the one proposed in [46]. As the Fitts' 
ID can be derived from an "aiming is choosing" rationale [42], we derived the temporal ID from a "waiting is choosing" rationale, which allows a generalization of the temporal ID to any compatible temporal task without relying on any assumption on the response function. We also corrected those ID multiplying them for the respective success rate (reaching or holding) [42]. Such ID therefore allows to consider not only reaching tasks, but also holding tasks, and could be hypothetically extended to more complex task composed by multiple reaching and holding phases to evaluate an invidual performance based on the average information transmitted in each phase. Through this framework, we found that, when considering only the reaching phase, the smallest target size is not always the one providing the highest information transmitted because the gain in source information associated with smaller targets may be overcome by the loss in transmission performance corresponding to a decrease in reaching success rate. When considering both reaching and holding phases, we found that spatial and temporal requirements affect each other, generally reducing a participants' optimal target size with respect to the reaching phase only, with the maximum information transmitted resulting for a specific, individual combination of spatial and temporal parameters. Such an approach could allow to hypothetically optimize an interface (not necessarily based on myoelectric control) depending on the user's capabilities, possibly also adapting the interface parameters as the user learns to control the device.

The prolonged usage of both prostheses and augmenting devices may have effects at the neural level. Amputation causes reorganization in the primary somatosensory cortex [77]. A recent study has showed that, in BCI control of independent DoFs, it is possible to dissociate neural gamma activity correlated to muscle activations [70]. Another study has shown that users of a third thumb controlled through a toe presented, after 5 days, a different representation of their hand in the sensorimotor cortex [78]. Considering the findings of such studies, we expect that even the intrinsic muscular null space control of an external device could bring some modifications in neural motor circuits. The exploitation of musculoskeletal redundancy to control a device is actually a new motor skill that requires learning, as it has been shown by the success rate curves from our study, and it is something different from the natural modulation of limb impedance [39] and even from the tele-impedance, which is based on the use of muscular null space to control the impedance of robots, providing them with a task-related elastic profile in addition to position trajectories [37], while no actual additional DoF is controlled. Thus, we hypothesize that, in this context, null space control improvements with training could be associated to the acquisition of novel muscular null space synergies, possibly encoded in the corticospinal pathways [79] and in the cortico-cerebellar circuits [80]. Investigation of the neuroplasticity associated to learning null space control may be necessary to test such a hypothesis.

Another important finding that has been reported in the literature is that, as for skill learning [81-83], feedback mechanisms integrated in an interface could help users improving their performance faster and to a higher level. It has been demonstrated that somatosensory feedback facilitates to learn controlling both prostheses [84] and augmenting devices [85]. For this reason, we believe that integrating somatosensory feedback in wearable bi-directional interfaces based on intrinsic muscular null space control will improve performance and facilitate learning.

In conclusion, we demonstrated the feasibility of a novel approach to control extra DoFs using muscular null space signals from many muscles directly involved in a task being performed concurrently. Participants in our experiment were able to reach targets and their performances improved with practice. Such an approach could be applied to control more sophisticated assistive or augmentative robotic devices (as extra limbs) in everyday life situations, for both able-bodied and disable-bodied people. Such approach is substantially different from the myoelectric control of exoskeletons, as they do not add additional DoFs [7,10,21]. We also developed an assessment framework based on information theory inspired by Fitts' law, with two indices of difficulty, which could be useful to quantify a participant's ability in reaching and holding a position independently from specific parameters of the assessment task. Further work is needed to understand the neural origin and mechanisms underlying learning of null space control. These results can be a starting point for the investigation of muscular null space control for augmentation, and our information theory approach can provide a novel tool to assess the ability of individual participants to control a device through noisy signals such as EMG, considering not only spatial precision, but also temporal precision.

\section{References}

[1] Fougner A, Stavdahl Ø, Kyberd P J, Losier Y G and Parker P A 2012 Control of Upper Limb Prostheses: Terminology and Proportional Myoelectric Control-A Review IEEE Transactions on Neural Systems and Rehabilitation Engineering 20 663-77

[2] Ha K H, Varol H A and Goldfarb M 2011 Volitional control of a prosthetic knee using surface electromyography IEEE Transactions on Biomedical Engineering 58 144-51

[3] Huang S, Wensman J P and Ferris D P 2014 An experimental powered lower limb prosthesis using proportional myoelectric control Journal of Medical Devices, Transactions of the ASME $\mathbf{8}$

[4] Jiang N, Rehbaum H, Vujaklija I, Graimann B and Farina D 2014 Intuitive, Online, Simultaneous, and Proportional Myoelectric Control Over Two Degrees-of-Freedom in 
Upper Limb Amputees IEEE Trans. Neural Syst. Rehabil. Eng. 22 501-10

[5] Parker P A and Scott R N 1986 Myoelectric control of prostheses Crit Rev Biomed Eng 13 283-310

[6] Vujaklija I, Farina D and Aszmann O 2016 New developments in prosthetic arm systems ORR Volume 831 9

[7] Dollar A M and Herr H 2008 Lower extremity exoskeletons and active orthoses: Challenges and state-of-the-art IEEE Transactions on Robotics 24 144-58

[8] Ferris D P and Lewis C L 2009 Robotic lower limb exoskeletons using proportional myoelectric control 2009 Annual International Conference of the IEEE Engineering in Medicine and Biology Society 2009 Annual International Conference of the IEEE Engineering in Medicine and Biology Society pp 2119-24

[9] Lunardini F, Casellato C, d'Avella A, Sanger T D and Pedrocchi A 2016 Robustness and Reliability of SynergyBased Myocontrol of a Multiple Degree of Freedom Robotic Arm IEEE Transactions on Neural Systems and Rehabilitation Engineering 24 940-50

[10] Singh R M and Chatterji S 2012 Trends and Challenges in EMG Based Control Scheme of Exoskeleton Robots - A Review International Journal of Scientific and Engineering Research 3 1-8

[11] Song R, Tong K, Hu X and Zhou W 2013 Myoelectrically controlled wrist robot for stroke rehabilitation Journal of NeuroEngineering and Rehabilitation 1052

[12] Berger D J, Gentner R, Edmunds T, Pai D K and d'Avella A 2013 Differences in Adaptation Rates after Virtual Surgeries Provide Direct Evidence for Modularity J. Neurosci. 33 12384-94

[13] Rugy A de, Loeb G E and Carroll T J 2012 Muscle Coordination Is Habitual Rather than Optimal J. Neurosci. 32 7384-91

[14] Barnard A, Jackson A and Jackson A 2012 Flexible Cortical Control of Task-Specific Muscle Synergies J. Neurosci. 32 12349-60

[15] Radhakrishnan S M, Baker S N and Jackson A 2008 Learning a Novel Myoelectric-Controlled Interface Task Journal of Neurophysiology 100 2397-408

[16] Sang-Hui Park and Seok-Pil Lee 1998 EMG pattern recognition based on artificial intelligence techniques IEEE Transactions on Rehabilitation Engineering 6 400-5

[17] Young A J, Smith L H, Rouse E J and Hargrove L J 2013 Classification of Simultaneous Movements Using Surface EMG Pattern Recognition IEEE Transactions on Biomedical Engineering 60 1250-8
[18] Amsuess S, Goebel P, Graimann B and Farina D 2015 A Multi-Class Proportional Myocontrol Algorithm for Upper Limb Prosthesis Control: Validation in Real-Life Scenarios on Amputees IEEE Trans. Neural Syst. Rehabil. Eng. 23 $827-36$

[19] Jiang N, Englehart K B and Parker P A 2009 Extracting Simultaneous and Proportional Neural Control Information for Multiple-DOF Prostheses From the Surface Electromyographic Signal IEEE Trans. Biomed. Eng. $\mathbf{5 6}$ $1070-80$

[20] Vukobratovic M, Borovac B, Surla D and Stokic D 2012 Biped Locomotion: Dynamics, Stability, Control and Application (Springer Science \& Business Media)

[21] Fleischer C and Hommel G 2008 A Human-Exoskeleton Interface Utilizing Electromyography IEEE Transactions on Robotics 24 872-82

[22] Johnson D C, Repperger D W and Thompson G 1996 Development of a mobility assist for the paralyzed, amputee, and spastic patient Proceedings of the 1996 Fifteenth Southern Biomedical Engineering Conference Proceedings of the 1996 Fifteenth Southern Biomedical Engineering Conference pp 67-70

[23] Ambrosini E, Ferrante S, Schauer T, Klauer C, Gaffuri M, Ferrigno G and Pedrocchi A 2014 A myocontrolled neuroprosthesis integrated with a passive exoskeleton to support upper limb activities Journal of Electromyography and Kinesiology 24 307-17

[24] Stein J, Narendran K, McBean J, Krebs K and Hughes R 2007 Electromyography-Controlled Exoskeletal UpperLimb-Powered Orthosis for Exercise Training After Stroke American Journal of Physical Medicine \& Rehabilitation $\mathbf{8 6}$ 255-61

[25] Colombo G, Joerg M, Schreier R and Dietz V 2000 Treadmill training of paraplegic patients using a robotic orthosis Journal of rehabilitation research and development 37 693-700

[26] Dominijanni G, Shokur S, Salvietti G, Buehler S, Palmerini E, Rossi S, De Vignemont F, D'Avella A, Makin T R, Prattichizzo D and Micera S 2021 Enhancing human bodies with extra robotic arms and fingers: The Neural Resource Allocation Problem arXiv:2103.17252 [cs, eess]

[27] Abdi E, Burdet E, Bouri M, Himidan S and Bleuler H 2016 In a demanding task, three-handed manipulation is preferred to two-handed manipulation Sci Rep 621758

[28] Kieliba P, Clode D, Maimon-Mor R O and Makin T R 2020 Neurocognitive consequences of hand augmentation bioRxiv 2020.06.16.151944

[29] Baldi T L, D'Aurizio N, Gaudeni C, Gurgone S, Borzelli D, D'Avella A and Prattichizzo D 2020 Exploiting Implicit Kinematic Kernel for Controlling a Wearable Robotic Extrafinger arXiv:2012.03600 [cs] 
[30] Prattichizzo D, Malvezzi M, Hussain I and Salvietti G 2014 The Sixth-Finger: A modular extra-finger to enhance human hand capabilities The 23rd IEEE International Symposium on Robot and Human Interactive Communication The 23rd IEEE International Symposium on Robot and Human Interactive Communication pp 993-8

[31] Salvietti G, Hussain I, Cioncoloni D, Taddei S, Rossi S and Prattichizzo D 2017 Compensating Hand Function in Chronic Stroke Patients Through the Robotic Sixth Finger IEEE Transactions on Neural Systems and Rehabilitation Engineering 25 142-50

[32] Parietti F and Asada H H 2017 Independent, voluntary control of extra robotic limbs 2017 IEEE International Conference on Robotics and Automation (ICRA) 2017 IEEE International Conference on Robotics and Automation (ICRA) pp 5954-61

[33] Penaloza C I and Nishio S 2018 BMI control of a third arm for multitasking Science Robotics 3 1-6

[34] Burdet E, Osu R, Franklin D W, Milner T E and Kawato M 2001 The central nervous system stabilizes unstable dynamics by learning optimal impedance Nature 414 446-9

[35] Milner T E 2002 Adaptation to destabilizing dynamics by means of muscle cocontraction Exp Brain Res 143 406-16

[36] Selen L P J, Franklin D W and Wolpert D M 2009 Impedance Control Reduces Instability That Arises from Motor Noise J. Neurosci. 29 12606-16

[37] Ajoudani A, Tsagarakis N G and Bicchi A 2012 Teleimpedance: Towards transferring human impedance regulation skills to robots 2012 IEEE International Conference on Robotics and Automation 2012 IEEE International Conference on Robotics and Automation pp $382-8$

[38] Laghi M, Ajoudani A, Catalano M G and Bicchi A 2020 Unifying bilateral teleoperation and tele-impedance for enhanced user experience The International Journal of Robotics Research 39 514-39

[39] Borzelli D, Cesqui B, Berger D J, Burdet E and d'Avella A 2018 Muscle patterns underlying voluntary modulation of co-contraction PLOS ONE 13 e0205911

[40] Takagi A, Kambara H and Koike Y 2020 Independent control of cocontraction and reciprocal activity during goaldirected reaching in muscle space Sci Rep $\mathbf{1 0} 22333$

[41] Bräcklein M, Ibáñez J, Barsakcioglu D Y and Farina D 2021 Towards human motor augmentation by voluntary decoupling beta activity in the neural drive to muscle and force production J. Neural Eng. 18016001

[42] Gori J, Rioul O and Guiard Y 2018 Speed-Accuracy Tradeoff: A Formal Information-Theoretic Transmission Scheme (FITTS) ACM Trans. Comput.-Hum. Interact. 25 $27: 1-27: 33$
[43] Kamavuako E N, Scheme E J and Englehart K B 2014 On the usability of intramuscular EMG for prosthetic control: A Fitts' Law approach Journal of Electromyography and Kinesiology 24 770-7

[44] Soukoreff R W and MacKenzie I S 2009 An informatic rationale for the speed-accuracy trade-off 2009 IEEE International Conference on Systems, Man and Cybernetics 2009 IEEE International Conference on Systems, Man and Cybernetics - SMC (San Antonio, TX, USA: IEEE) pp 2890-6

[45] Soukoreff R W and MacKenzie I S 2004 Towards a standard for pointing device evaluation, perspectives on 27 years of Fitts' law research in HCI International Journal of Human-Computer Studies 61 751-89

[46] Lee B and Oulasvirta A 2016 Modelling Error Rates in Temporal Pointing Proceedings of the 2016 CHI Conference on Human Factors in Computing Systems CHI '16 (San Jose, California, USA: Association for Computing Machinery) pp $1857-68$

[47] Berger D J and d'Avella A 2014 Effective force control by muscle synergies Front. Comput. Neurosci. 8

[48] Hermens H J, Freriks B, Merletti R, Stegeman D, Blok J, Rau G, Disselhorst-Klug C and Hägg G European Recommendations for Surface ElectroMyoGraphy 4

[49] Fitts P M 1954 The information capacity of the human motor system in controlling the amplitude of movement Journal of Experimental Psychology 47 381-91

[50] MacKenzie I S 1992 Fitts' Law as a Research and Design Tool in Human-Computer Interaction Human-Computer Interaction 7 91-139

[51] Meyer D E, Keith-Smith J E, Kornblum S, Abrams R A and Wright C E 1990 Speed-accuracy tradeoffs in aimed movements: Toward a theory of rapid voluntary action Attention and performance 13: Motor representation and control (Hillsdale, NJ, US: Lawrence Erlbaum Associates, Inc) pp 173-226

[52] Newell A 1994 Unified Theories of Cognition (Harvard University Press)

[53] Carlton L G 1994 The Effects of Temporal-Precision and Time-Minimization Constraints on the Spatial and Temporal Accuracy of Aimed Hand Movements Journal of Motor Behavior 26 43-50

[54] Zelaznik H N, Mone S, McCabe G P and Thaman C 1988 Role of temporal and spatial precision in determining the nature of the speed-accuracy trade-off in aimed-hand movements Journal of Experimental Psychology: Human Perception and Performance 14 221-30

[56] Cha Y and Myung R 2013 Extended Fitts' law for 3D pointing tasks using 3D target arrangements International Journal of Industrial Ergonomics 43 350-5 
[57] Murata A and Iwase H 2001 Extending Fitts' law to a threedimensional pointing task Human Movement Science 20 791-805

[58] Welford A T 1968 Fundamentals of skill (New York, NY, US: Methuen)

[59] Welford A T 1960 The measurement of sensory-motor performance: Survey and reappraisal of twelve years' progress Ergonomics 3 189-230

[60] Reis J, Schambra H M, Cohen L G, Buch E R, Fritsch B, Zarahn E, Celnik P A and Krakauer J W 2009 Noninvasive cortical stimulation enhances motor skill acquisition over multiple days through an effect on consolidation PNAS 106 $1590-5$

[61] Shmuelof L, Krakauer J W and Mazzoni P 2012 How is a motor skill learned? Change and invariance at the levels of task success and trajectory control Journal of Neurophysiology 108 578-94

[62] Makin T R, de Vignemont F and Faisal A A 2017 Neurocognitive barriers to the embodiment of technology Nature Biomedical Engineering 1 1-3

[63] Abdi E, Burdet E, Bouri M and Bleuler H 2015 Control of a Supernumerary Robotic Hand by Foot: An Experimental Study in Virtual Reality PLOS ONE 10 e 0134501

[64] Noccaro A, Eden J, Di Pino G, Formica D and Burdet E 2021 Human performance in three-hands tasks Sci Rep 11 9511

[65] Saraiji M Y, Sasaki T, Kunze K, Minamizawa K and Inami M 2018 MetaArms: Body Remapping Using Feet-Controlled Artificial Arms The 31st Annual ACM Symposium on User Interface Software and Technology - UIST '18 The 31st Annual ACM Symposium (Berlin, Germany: ACM Press) pp $65-74$

[66] Salvietti G, Hussain I, Cioncoloni D, Taddei S, Rossi S and Prattichizzo D 2017 Compensating Hand Function in Chronic Stroke Patients Through the Robotic Sixth Finger IEEE Transactions on Neural Systems and Rehabilitation Engineering 25 142-50

[67] Guggenheim J W, Parietti F, Flash T and Asada H H 2020 Laying the Groundwork for Intra-Robotic-Natural Limb Coordination: Is Fully Manual Control Viable? J. Hum.Robot Interact. 9 18:1-18:12

[68] Parietti F and Asada H H 2017 Independent, voluntary control of extra robotic limbs 2017 IEEE International Conference on Robotics and Automation (ICRA) 2017 IEEE International Conference on Robotics and Automation (ICRA) (Singapore, Singapore: IEEE) pp 5954-61

[69] Guggenheim J, Hoffman R, Song H and Asada H H 2020 Leveraging the Human Operator in the Design and Control of Supernumerary Robotic Limbs IEEE Robotics and Automation Letters 5 2177-84
[70] Bashford L, Wu J, Sarma D, Collins K, Rao R P N, Ojemann J G and Mehring C 2018 Concurrent control of a brain-computer interface and natural overt movements $J$. Neural Eng. 15066021

[71] Milovanovic I, Robinson R, Fetz E E and Moritz C T 2015 Simultaneous and independent control of a brain-computer interface and contralateral limb movement Brain-Computer Interfaces 2 174-85

[72] Hoffmann E R 1991 Capture of moving targets: a modification of Fitts' Law Ergonomics 34 211-20

[73] Borish C N, Bertucco M and Sanger T D 2020 Effect of target distance on controllability for myocontrol International Journal of Human-Computer Studies 140 102432

[74] Mendez V, Iberite F, Shokur S and Micera S 2021 Current Solutions and Future Trends for Robotic Prosthetic Hands Annu. Rev. Control Robot. Auton. Syst. 4 595-627

[75] Card S K, English W K and Burr B J 1978 Evaluation of Mouse, Rate-Controlled Isometric Joystick, Step Keys, and Text Keys for Text Selection on a CRT Ergonomics 21 60113

[76] Crossman E 1957 The speed and accuracy of simple hand movements The nature and acquisition of industrial skills

[77] Makin T R and Flor H 2020 Brain (re)organisation following amputation: Implications for phantom limb pain NeuroImage 218116943

[78] Kieliba P, Clode D, Maimon-Mor R O and Makin T R 2021 Robotic hand augmentation drives changes in neural body representation Science Robotics 6

[79] Overduin S A, d'Avella A, Carmena J M and Bizzi E 2012 Microstimulation Activates a Handful of Muscle Synergies Neuron 76 1071-7

[80] Berger D J, Masciullo M, Molinari M, Lacquaniti F and d'Avella A 2020 Does the cerebellum shape the spatiotemporal organization of muscle patterns? Insights from subjects with cerebellar ataxias Journal of Neurophysiology 123 1691-710

[81] Morris D, Tan H, Barbagli F, Chang T and Salisbury K 2007 Haptic Feedback Enhances Force Skill Learning Second Joint EuroHaptics Conference and Symposium on Haptic Interfaces for Virtual Environment and Teleoperator Systems (WHC'07) Second Joint EuroHaptics Conference and Symposium on Haptic Interfaces for Virtual Environment and Teleoperator Systems (WHC'07) pp 21-6

[82] Wolpert D M, Miall R C and Kawato M 1998 Internal models in the cerebellum Trends in Cognitive Sciences $\mathbf{2}$ $338-47$ 
[83] Wulf G, Shea C H and Matschiner S 1998 Frequent Feedback Enhances Complex Motor Skill Learning Journal of Motor Behavior 30 180-92

[84] Stepp C E, An Q and Matsuoka Y 2012 Repeated training with augmentative vibrotactile feedback increases object manipulation performance PLOS ONE 7

[85] Amoruso E, Dowdall L, Kollamkulam M T, Ukaegbu O, Kieliba P, Ng T, Dempsey-Jones H, Clode D and Makin T R 2021 Somatosensory signals from the controllers of an extra robotic finger support motor learning bioRxiv 2021.05.18.444661 